Article

\title{
Accumulation of Phosphorus-Containing Compounds in Developing Seeds of Low-Phytate Pea (Pisum sativum L.) Mutants
}

\author{
Arun S.K. Shunmugam 1, Cheryl Bock ${ }^{2}$, Gene C. Arganosa 1, Fawzy Georges ${ }^{2}$, \\ Gordon R. Gray ${ }^{1}$ and Thomas D. Warkentin ${ }^{1, *}$
}

1 Crop Development Centre, Department of Plant Sciences, University of Saskatchewan, 51 Campus Drive, Saskatoon, SK S7N 5A8, Canada; E-Mails: s.ask@usask.ca (A.S.K.S.); gene.arganosa@usask.ca (G.C.A.); gr.gray@usask.ca (G.R.G.)

2 National Research Council Canada, 110 Gymnasium Place, Saskatoon, SK S7N 0W9, Canada; E-Mails: Cheryl.bock@nrc-cnrc.gc.ca (C.B.); fawzy.georges@usask.ca (F.G.)

* Author to whom correspondence should be addressed; E-Mail: tom.warkentin@usask.ca; Tel.: +1-306-966-2371.

Academic Editor: Francesca Sparvoli

Received: 24 October 2014 / Accepted: 17 December 2014 / Published: 26 December 2014

\begin{abstract}
Low phytic acid (lpa) crops are low in phytic acid and high in inorganic phosphorus $\left(\mathrm{P}_{\mathrm{i}}\right)$. In this study, two lpa pea genotypes, 1-150-81, 1-2347-144, and their progenitor CDC Bronco were grown in field trials for two years. The lpa genotypes were lower in IP6 and higher in Pi when compared to CDC Bronco. The total P concentration was similar in lpa genotypes and CDC Bronco throughout the seed development. The action of myo-inositol phosphate synthase (MIPS) (EC 5.5.1.4) is the first and rate-limiting step in the phytic acid biosynthesis pathway. Aiming at understanding the genetic basis of the lpa mutation in the pea, a $1530 \mathrm{bp}$ open reading frame of MIPS was amplified from CDC Bronco and the lpa genotypes. Sequencing results showed no difference in coding sequence in MIPS between CDC Bronco and lpa genotypes. Transcription levels of MIPS were relatively lower at 49 days after flowering (DAF) than at 14 DAF for CDC Bronco and lpa lines. This study elucidated the rate and accumulation of phosphorus compounds in lpa genotypes. The data also demonstrated that mutation in MIPS was not responsible for the lpa trait in these pea lines.
\end{abstract}


Keywords: D-myo-inositol phosphate synthase (MIPS); phosphorus; phytate biosynthesis; phytic acid

\section{Introduction}

Phytic acid (myo-inositol-1,2,3,4,5,6-hexakisphosphate; $\mathrm{IP}_{6}$ ) is the major storage form of phosphorus (P) in most plant seeds [1]. Phytate is present within subcellular protein inclusions in all seeds. Particularly, in cereal grains, phytates are essentially localized in the germ and aleurone tissues whereas in dicotyledons, phytate is distributed throughout the cotyledon [2,3]. Endogenous phytase enzymes break down phytate during seed germination and release its phosphorus, myo-inositol, and mineral contents for use by the growing seedling [4]. IP 6 also accumulates in other plant tissues and organs that accumulate nutrient stores for subsequent redistribution, such as pollen, roots, and tubers [5].

Applied interest in seed $\mathrm{IP}_{6}$ primarily concerns its roles in human health and animal nutrition. It is a strong chelator of mineral elements such as iron, zinc, calcium, and potassium, forming mixed salts that are largely excreted by humans and other non-ruminant animals such as poultry, swine, and fish [6,7]. Excretion of seed-derived $\mathrm{IP}_{6}$ can contribute to dietary iron and zinc deficiencies, a major public health problem in the developing world. In addition, undigested phytate excreted by non-ruminant animals represents an important source of phosphorus pollution in the environment [8]. Due to nutritional and environmental concerns, the development of cultivars with a low-phytate trait has become an attractive breeding objective in many crop species. Chemically induced, non-lethal recessive mutants that decrease seed phytic acid content have been isolated and genetically mapped in maize (Zea mays L.) [9,10], barley (Hordeum vulgare L.) [11,12], and soybean (Glycine max L. Merr) [13]. Recently, Warkentin et al. [14] developed and characterized two low-phytic acid mutants of field pea (Pisum sativum L.). The low phytic acid (lpa) mutations have the potential to alleviate the environmental and nutritional problems associated with phytic acid in animal feeds [15]. Moreover, lpa crops may also offer improved nutrition for human populations that depend upon grains and legumes as staple foods. In addition, these lpa mutants provide a valuable system to study seed phytic acid synthesis.

In plants, the six-carbon cyclitol myo-inositol gives rise to compounds with roles in such diverse functions as signal transduction, membrane biogenesis, stress tolerance, and the generation of seed storage compounds including $\mathrm{IP}_{6}[16,17]$. The de novo synthesis of myo-inositol involves the conversion of glucose 6-phosphate to myo-inositol-1-phosphate (IP $\left.{ }_{1}\right)$ that is subsequently dephosphorylated to release free myo-inositol. The former reaction is catalyzed by myo-inositol-1-phosphate synthase (MIPS; EC 5.5.1.4), the first and rate-limiting enzyme of the pathway [17-19]. This makes MIPS an attractive target for manipulation to produce low-phytate crops. Previous studies have demonstrated that $50 \%$ to $95 \%$ reductions in phytic acid can be obtained when this enzyme is targeted through mutagenesis or by genetic engineering methodologies [20-26]. In soybean, mutations in MIPS coding sequences conferred a decreased phytic acid phenotype, and an effective reduction in phytate content ( $90 \%$ to $95 \%$ ) has been observed when one of the four MIPS in soybean, GmMIPS1 was silenced through a RNA interference (RNAi) approach [24,26]. An lpa phenotype was also produced by manipulating the MIPS gene through an antisense approach in Oryza sativa L. [22]. 
The objective of this study was to investigate the accumulation of phytic acid and other phosphorus compounds in developing seeds of normal and low-phytate genotypes of field pea (Pisum sativum L.). We also examined MIPS gene expression and analyzed the sequence at the nucleotide and protein levels to ascertain if variation in MIPS coding sequences was responsible for the lpa trait. This will help us to understand the nature of the low phytate mutation(s) and develop markers for the low phytate trait furthering the development of low-phytate cultivars.

\section{Results}

\subsection{Agronomic Characteristics of Low-Phytate Pea Genotypes}

The agronomic characteristics of the lpa pea genotypes were similar to their normal phytate progenitor CDC Bronco except for 1000 seed weight and grain yield at maturity (Figure 1, Table 1). CDC Bronco and genotypes 1-150-81 and 1-2347-144 did not differ in percent emergence, days to flowering, plant height, mycosphaerella blight score, lodging and days to maturity at all four site years (Table 1). CDC Bronco was higher in mean 1000 seed weight (219 g) than 1-150-81 and 1-2347-144 with 207 and $205 \mathrm{~g}$, respectively. CDC Bronco had the highest grain yield (2.83 $\left.\mathrm{t} \cdot \mathrm{ha}^{-1}\right)$, significantly greater than genotypes 1-150-81 and 1-2347-144 with 2.36 and $2.33 \mathrm{t} \cdot \mathrm{ha}^{-1}$, respectively.

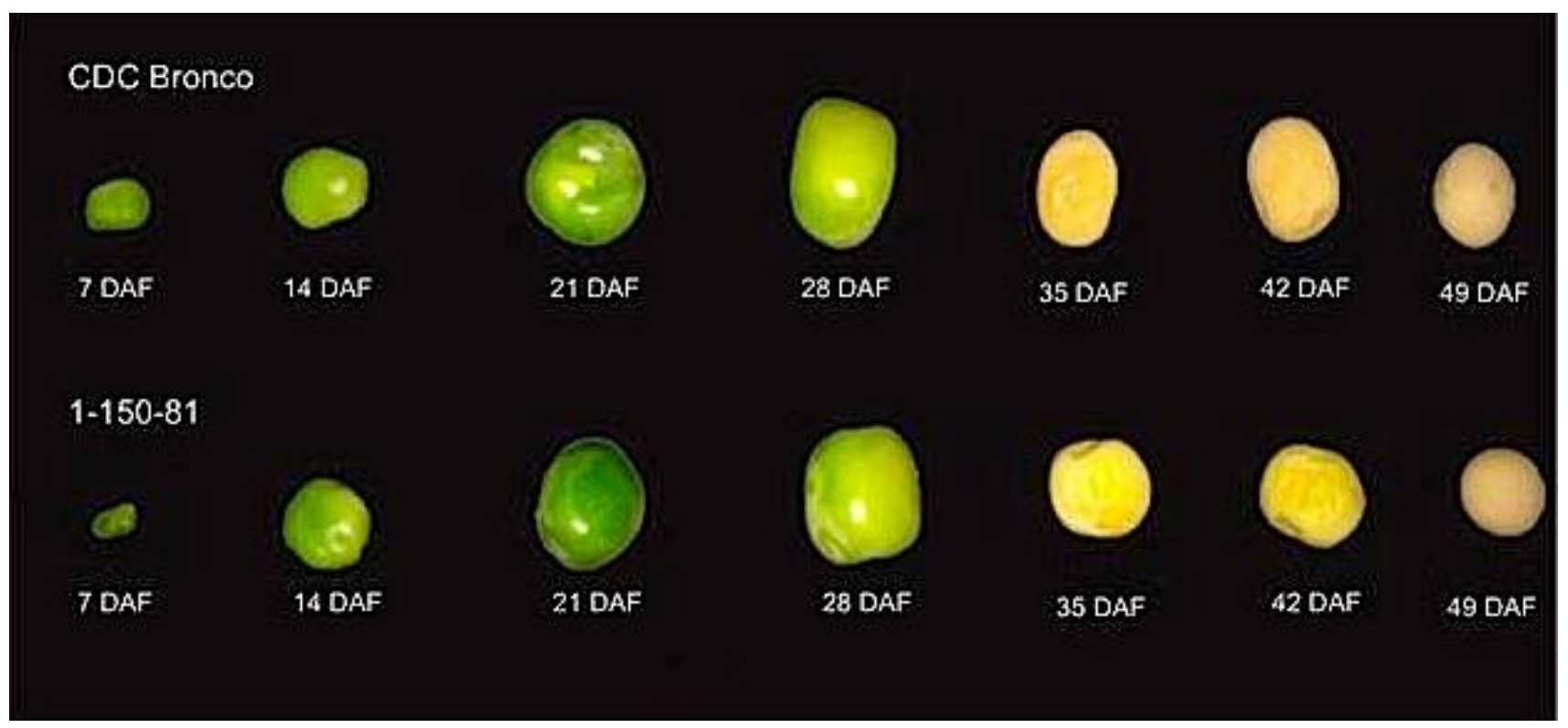

Figure 1. Developing seeds of pea (Pisum sativum L.). Indicated are CDC Bronco and low-phytate genotype 1-150-81. Representative photographs are shown from the Rosthern site in 2010. DAF stands for "days after flowering".

\subsection{Accumulation of Phosphorus and Phosphorus-Containing Compounds during Seed Development}

In seed coat extracts of CDC Bronco, 1-150-81 and 1-2347-144, there was no detectable amount of $\mathrm{IP}_{6}$ or other inositol polyphosphates during seed development from 14 to 49 DAF (data not shown [27]). In addition, no lower inositol phosphate intermediates $\left(\mathrm{IP}_{1}, \mathrm{IP}_{3}, \mathrm{IP}_{4}, \mathrm{IP}_{5}\right)$ other than phytic acid were detected in cotyledons of CDC Bronco, 1-150-81, and 1-2347-144 in any of the developmental stages analyzed (data not shown [27]). 
Table 1. Agronomic traits of pea (Pisum sativum L.) cultivar CDC Bronco and low-phytate genotypes 1-150-81 and 1-2347-144 assessed at Saskatoon and Rosthern, Saskatchewan in 2010 and 2011. Values represent means \pm SE, $n=16$. Different letters within a column indicate a significant difference at $p<0.05$ based on LSD test.

\begin{tabular}{|c|c|c|c|c|c|c|c|c|}
\hline Genotype & $\begin{array}{l}\text { Emergence } \\
\text { Count }(\%)^{d}\end{array}$ & Days to Flower & $\begin{array}{c}\text { Plant } \\
\text { Height }(\mathbf{c m})^{\mathrm{e}}\end{array}$ & $\begin{array}{c}\text { Mycosphaerella Blight } \\
\text { Score (0-9 Scale) }{ }^{\mathrm{f}} \\
\end{array}$ & $\begin{array}{c}\text { Lodging Score } \\
(1-9 \text { Scale })^{\mathrm{g}}\end{array}$ & Days to Mature & $\begin{array}{c}\text { Grain } \\
\text { Yield }\left(\mathrm{t} \cdot \mathrm{ha}^{-1}\right)^{\mathrm{h}} \\
\end{array}$ & $\begin{array}{l}1000 \text { Seed } \\
\text { Weight }(\mathrm{g})\end{array}$ \\
\hline CDC Bronco & $56 \pm 3.0^{\mathrm{a}}$ & $58 \pm 1.0^{\mathrm{a}}$ & $79 \pm 1.0^{\mathrm{a}}$ & $5.3 \pm 0.1^{\mathrm{a}}$ & $5.4 \pm 0.2^{\mathrm{a}}$ & $100 \pm 1.0^{\mathrm{a}}$ & $2.83 \pm 0.1^{\mathrm{a}}$ & $219 \pm 2.2^{\mathrm{a}}$ \\
\hline 1-150-81 & $55 \pm 3.0^{\mathrm{a}}$ & $59 \pm 1.0^{\mathrm{a}}$ & $78 \pm 2.0^{\mathrm{a}}$ & $5.2 \pm 0.1^{\mathrm{a}}$ & $5.3 \pm 0.1^{\mathrm{a}}$ & $102 \pm 1.0^{\mathrm{a}}$ & $2.36 \pm 0.2^{b}$ & $207 \pm 1.6^{b}$ \\
\hline $1-2347-144$ & $53 \pm 3.0^{\mathrm{a}}$ & $59 \pm 1.0^{\mathrm{a}}$ & $76 \pm 2.0^{\mathrm{a}}$ & $5.3 \pm 0.1^{\mathrm{a}}$ & $5.3 \pm 0.1^{\mathrm{a}}$ & $101 \pm 1.0^{\mathrm{a}}$ & $2.33 \pm 0.2^{b}$ & $205 \pm 2.3^{b}$ \\
\hline
\end{tabular}

${ }^{\mathrm{d}}$ Based on seedlings in a $1 \mathrm{~m}^{2}$ section of each plot counted 5 weeks after planting; ${ }^{\mathrm{e}}$ Measured when the pod set was completed; ${ }^{\mathrm{f}}$ Assessed base on $0-9$ scale, where $0=$ no disease, 9 = completely blighted; ${ }^{\mathrm{g}}$ Assessed based on 1-9 scale, where 1 = erect, $9=$ completely lodged; ${ }^{\mathrm{h}}$ Residual harvest weighed after sampling developing seeds (120 pods from each plot). 
The concentration of IP 6 at 14 DAF was not significantly different among CDC Bronco, 1-150-81, and 1-2347-144 (Figure 2a). However, the concentrations of IP6 among CDC Bronco, 1-150-81 and 1-2347-144 started to differ significantly from 21 DAF onwards (Figure 2a). In CDC Bronco, the concentration of IP 6 ranged from $0.20 \mathrm{mg} \cdot \mathrm{g}^{-1} \mathrm{DW}$ at $14 \mathrm{DAF}$ and increased steadily to $1.86 \mathrm{mg} \cdot \mathrm{g}^{-1} \mathrm{DW}$ at 49 DAF (Figure 2a). A similar trend was observed for IP 6 in 1-150-81 and 1-2347-144. However, the lpa genotypes 1-150-81 and 1-2347-144 showed 65\% and 60\% reduction in $\mathrm{IP}_{6}$, respectively, when compared to their progenitor CDC Bronco at 49 DAF (Figure 2a). In 1-150-81 IP 6 concentrations ranged from 0.11 to $0.65 \mathrm{mg} \cdot \mathrm{g}^{-1} \mathrm{DW}$ while the range of $\mathrm{IP}_{6}$ concentration in $1-2347-144$ was 0.08 to $0.75 \mathrm{mg} \cdot \mathrm{g}^{-1}$ DW (Figure 2a). Since it was assumed that all phytic acid-P ( $\mathrm{PphA}_{2}$ came from $\mathrm{IP}_{6}$, these results mirrored those of phytic acid. Absolute values are presented in Figure A1.

$\mathrm{P}_{\mathrm{i}}$ concentration at 14 DAF was not significantly different for CDC Bronco and 1-150-81 with 2.62 and $2.44 \mathrm{mg} \cdot \mathrm{g}^{-1}$ dry weight (DW), respectively (Figure 2b). At $14 \mathrm{DAF}, 1-2347-144$ had more $\mathrm{P}_{\mathrm{i}}$ (3.24 $\left.\mathrm{mg} \cdot \mathrm{g}^{-1} \mathrm{DW}\right)$ than the other two genotypes. From $21 \mathrm{DAF}$ to $49 \mathrm{DAF}, \mathrm{P}_{\mathrm{i}}$ concentrations between the lpa genotypes were similar and significantly higher than CDC Bronco (Figure 2b). At 49 DAF, 1-150-81 and 1-2347-144 were 72\% and 84\% higher in $\mathrm{P}_{\mathrm{i}}$, respectively, than CDC Bronco.

The total $\mathrm{P}$ accumulation pattern was similar between CDC Bronco and the two lpa genotypes (Figure 2c). The concentration of total $\mathrm{P}$ was not significantly different between CDC Bronco and the lpa genotypes except at 21 DAF. CDC Bronco had $3.44 \mathrm{mg} \cdot \mathrm{g}^{-1} \mathrm{DW}$ total $\mathrm{P}$ at $21 \mathrm{DAF}$ that was significantly different from 1-150-81 and 1-2347-144 with 3.16 and $3.36 \mathrm{mg} \cdot \mathrm{g}^{-1} \mathrm{DW}$ total $\mathrm{P}$, respectively (Figure $2 \mathrm{c}$ ). The variations in $\mathrm{IP}_{6}$ and $\mathrm{P}_{\mathrm{i}}$ levels did not affect the total $\mathrm{P}$ concentration of these genotypes.

\subsection{Characterization and Bioinformatic Analyses of PSMIPS}

The PSMIPS primers amplified a single product of the expected size (1602-bp) from 14 DAF seed samples of CDC Bronco, 1-150-81, and 1-2347-144 (data not shown [27]). These fragments were excised and sequenced. The obtained cDNA sequence contained a 1530-bp open reading frame (ORF), and encoded a protein of 510 amino acids with a molecular weight of $56.5 \mathrm{kD}$ and $\mathrm{pI}$ of 5.35 (Figure 3). The PsMIPS ORFs of CDC Bronco and the two lpa mutants, 1-150-81 and 1-2347-144, demonstrated a $100 \%$ homology (Figure A2). There were no mutations observed in the form of nucleic acid substitutions between the three ORFs.

The deduced amino acid sequence of PsMIPS was aligned along with four other plant MIPS and is shown in Figure 4. The MIPS protein from Pisum sativum has a 97\% identity with MIPS from Medicago truncatula, a 96\% identity with Cicer arietinum, a 94\% identity with Glycine max and a 92\% identity with MIPS from Phaseolus vulgaris (Figure 4). Also present in PsMIPS are four motifs that are highly conserved in all MIPS proteins: GWGGNNG (Domain 1), LWTANTERY (Domain 2), NGSPQNTFVPGL (Domain 3) and SYNHLGNNDG (Domain 4), all of which are involved in cofactor $\left(\mathrm{NAD}^{+}\right)$binding and reaction catalysis of MIPS protein (Figure 4) [28,29]. 
a

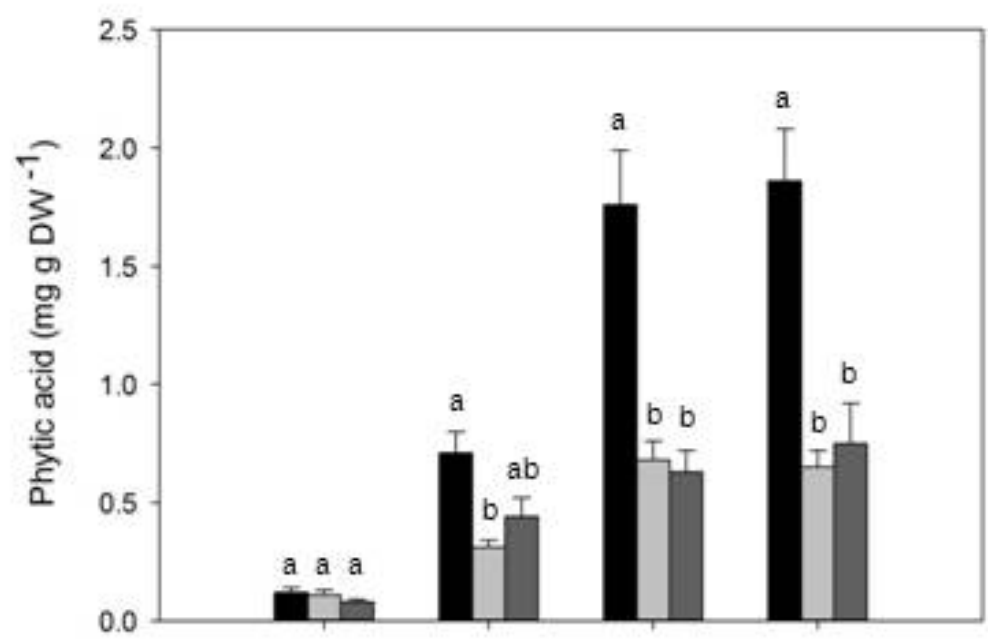

b

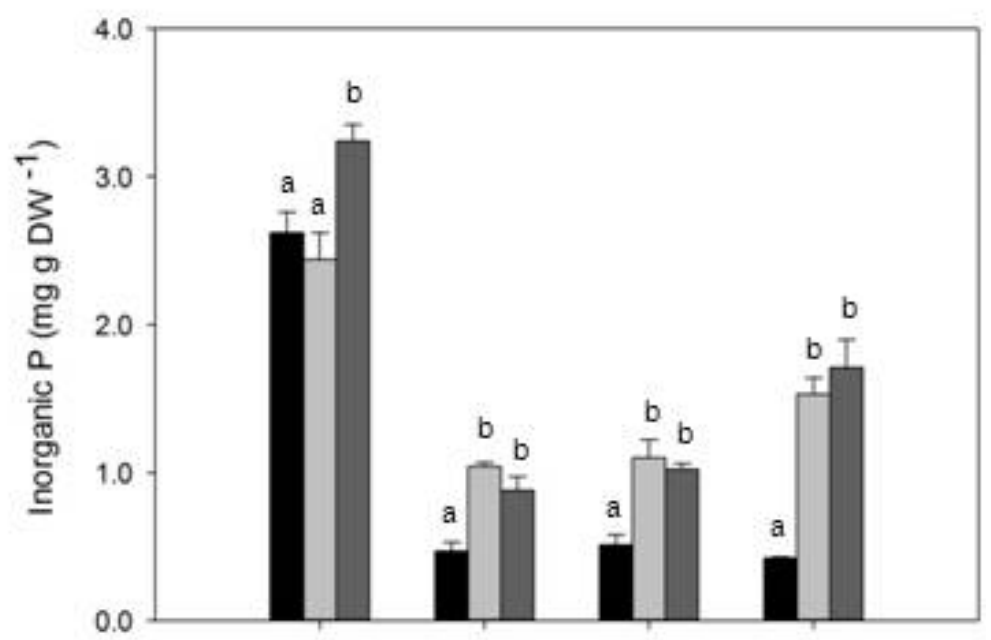

C

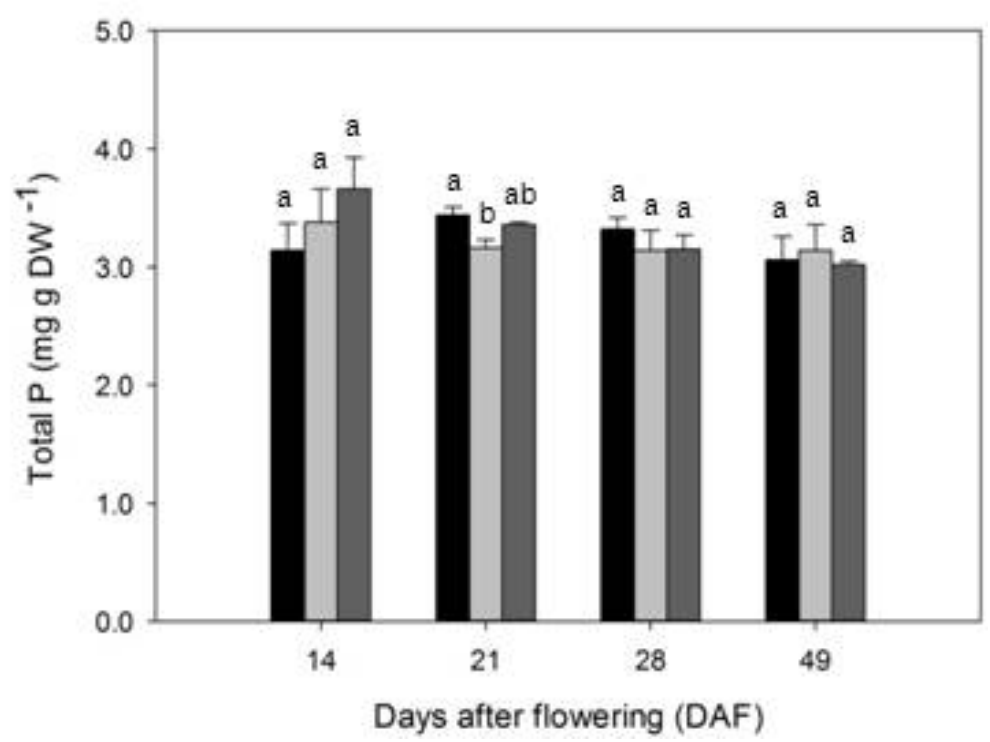

Figure 2. (a) Content of phytic acid; (b) Content of inorganic $\mathrm{P}\left(\mathrm{P}_{\mathrm{i}}\right)$; (c) Content of total $\mathrm{P}$ in developing seeds of pea (Pisum sativum L.) for CDC Bronco (black bars) and low-phytate genotypes 1-150-81 (light grey bars) and 1-2347-144 (dark grey bars) assessed at Saskatoon and Rosthern, Saskatchewan in 2010 and 2011. Values represent means \pm SE, $n=4$. Different letters associated with bars within each DAF are significantly different at $p<0.05$. DAF, days after flowering; DW, dry weight; P, phosphorus. 
cctttctctttgtgatttccatattcaccaaatgttcatagagagtttcaaggttgag M E I $E$ E $S$ F $K$ V $E$ agtcctaacgtgaagtacacagacacagagattcagtctgtgtacagttacgaaacaact

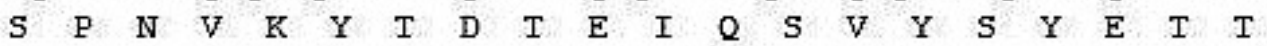
gaacttgttcatcagaacagaaatgacacttatcaatggattgttaaccctaaaactgtg $\begin{array}{lllllllllllllllllllll}E & \mathrm{~L} & \mathrm{~V} & \mathrm{H} & \mathrm{Q} & \mathrm{N} & \mathrm{R} & \mathrm{N} & \mathrm{D} & \mathrm{T} & \mathrm{Y} & \mathbf{Q} & \mathrm{W} & \mathrm{I} & \mathrm{V} & \mathrm{N} & \mathrm{P} & \mathrm{K} & \mathrm{T} & \mathrm{V}\end{array}$ aaatatgaatttaaaaccgaaactcatgttcctaaattgggggtaatgcttgtgggatgg $\begin{array}{lllllllllllllllllllll}\text { K } & Y & \text { E } & \text { F } & \text { K } & \text { T } & \text { E } & \text { T } & \text { H } & \text { V } & \text { P } & \text { K } & \text { L } & G & \text { V } & M & \text { L } & V & G & \text { W }\end{array}$ ggtggaaacaacggttcaacccttaccggtggtgttattgctaatcgagagggtatttca $\begin{array}{llllllllllllllllllll}G & G & N & N & G & S & I & L & T & G & G & V & I & A & N & R & E & G & I & S\end{array}$ tgggcaacgaaagataatattcaacaagcgaattactttggttctctcactcaagcttca

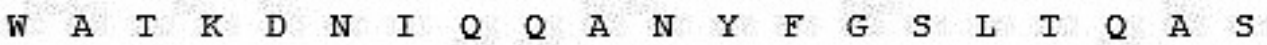
gctattcgtgttggatcttccaaggagaggaaattcatgctccctcaagagcttgtta

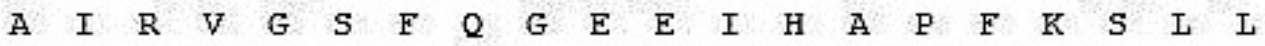
ccaatggtcaaccctgatgacattgtttttggtggatgggatatcagtaacatgaacctt $\begin{array}{llllllllllllllllllll}P & M & V & N & \text { P } & D & D & I & V & F & G & G & W & D & I & S & N & M & N & L\end{array}$ gctgatgccatggctagggccagggttttcgacattgatttgcaaaagcaattgaggcct

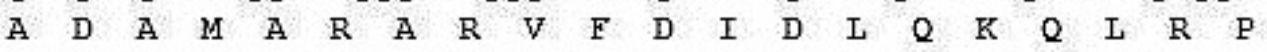
tatatggaatccatggttccactccccggcatctatgacccggatttcattgctgccaat

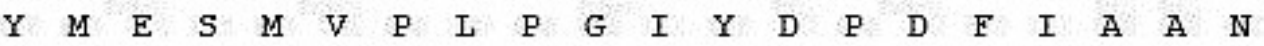
caaggtgaacgtgcaaataatgttattaagggtacaaagagagaacaaattaaccaaatc $\begin{array}{lllllllllllllllllllll} & Q & G & E & R & A & N & N & V & I & K & G & T & K & R & E & Q & I & N & Q & I\end{array}$ atcaaagacattcgggaatttaaggaagcaaacaaagtagacagggttgttgttctctgg $\begin{array}{lllllllllllllllllllll}I & K & D & I & R & E & F & K & E & A & N & K & V & D & R & V & V & V & L & W\end{array}$ actgccaacacagagaggtacagtaatttagttgtgggactcaatgacaccacagagaac

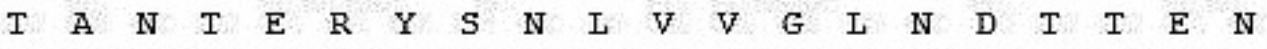
cttttgctgcagtggacagaaatgagtctgagatttctccttccaccctgtttggcatt $\begin{array}{llllllllllllllllllll}L & F & A & A & V & D & R & N & E & S & E & I & S & P & S & T & L & F & G & I\end{array}$ gcttgtgttatggaaalgttccttcatcaatggaagccctcagaacacttttgttcca $\begin{array}{lllllllllllllllllllll}A & C & V & M & E & N & V & P & F & I & N & G & S & P & Q & N & T & F & V & P\end{array}$ gggcttattgatcttgccatcaagaacaacaccttgattggtggcgatgacttcaagagt $\begin{array}{llllllllllllllllllll}G & L & I & D & L & A & I & K & N & N & T & L & I & G & G & D & D & F & K & S\end{array}$ ggtcagaccaaargaaatctgttttggttgatttccttgttggagctggtatcaagcca

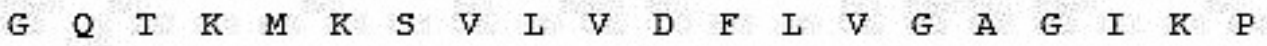
acgtcgatagtgagttacaatcatcttggaaacaatgatggtatgaacctctcagcacca

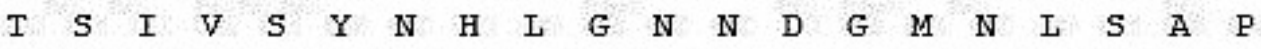
caaaccttccgctccaaggaaatctccaagagcaacgttgttgacgatatggtcaacagc

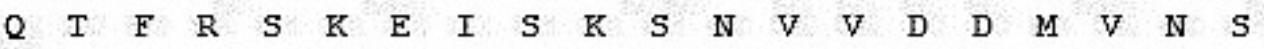
aacgctatcctctatgcgcctggtgaacatcctgaccatgttgtagtcattaagtatgtg

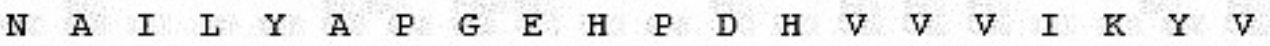
ccatacgtcggagacagcaagagagccatggacgagtatacttcggaaatattcatgggt $\begin{array}{llllllllllllllllllll}P & Y & V & G & D & S & K & R & A & M & D & E & Y & T & S & E & I & F & M & G\end{array}$ ggaaagaacactattgtgttgcacaacacatgtgaggattccctcttggctgcccctatt $\begin{array}{llllllllllllllllllll}G & K & N & I & I & V & L & H & N & \text { I } & C & E & D & S & L & L & A & A & P & I\end{array}$ atcttggacttggttcttcttgctgagcttagtactagaattcagttcaaatctgaagct

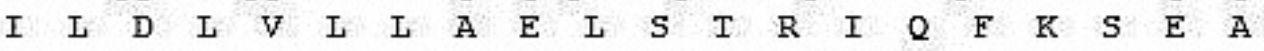
gagaacaagtttcacacattccaccctgttgctaccatcctcagttatctgaccaaggct

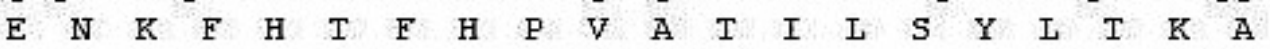
cctctggttccaccaggtacaccagtggtgaatgcattgtccaagcagcgagcgatgctg $\begin{array}{llllllllllllllllllll}P & L & V & P & P & G & I & P & V & V & N & A & L & S & K & Q & R & A & M & L\end{array}$ gaaacatcatgagagcttgtgttggattggctccagaaaacaacatgatccttgagtac $\begin{array}{lllllllllllllllllllll} & \mathrm{N} & \mathrm{N} & \mathrm{I} & \mathrm{M} & \mathrm{R} & \mathrm{A} & \mathrm{C} & \mathrm{V} & \mathrm{G} & \mathrm{L} & \mathrm{A} & \mathrm{P} & \mathrm{E} & \mathrm{N} & \mathrm{N} & \mathrm{M} & \mathrm{I} & \mathrm{L} & \mathrm{E} & \mathrm{Y}\end{array}$ aagtgaagcaggggatagaatcattagtgattaat

$\mathrm{K}-$

Figure 3. PsMIPS cDNA and deduced amino acid sequence from Pisum sativum CDC Bronco. Initiation and termination codons are boxed and shown in bold text. 

P. sativum
M. truncatula
C. arietinum
G. $\max$
P. vulgaris
P. sativum
M. truncatula
C. arietinum
G. $\max$
P. vulgaris

1 MF IESFKVES PNVKYTDTEI QSVYSYETTELVHQNRNDTYQWIVNPKTVKYE FKTE THVP

1 MF IENEKVESPNVKYTETEI QSVYNYETTELVHENRNGTYQWIVKPKTVKYE FKTDIHVP

1 MF IENFKVDSPNVKYTETEIQSVYNYETTELVHENRNGTYQWIVKPKTVKYEFKTDTHVP

1 MF IENEKVECPNVKYTETEI QSVYNYETTELVHENRNGTYQWIVKPKSVKYE FKTNIHVP

1 MF IENEKVESPNVKYSETEI QSVYNY ETTELVHENRNG M़् YQWI I KPKSVKYE FKTNTHVP

61 KL GVMLVGWGGNNGSTLTGGVIANRE GISWAT KDYI IQQANYFGS LTQASA IRVGSFQGEE 61 KL GVMLVGWGGNNGSTLTGGVIANRE GISWAT KDKIQQANYFGSLTQASA IRVGSFOGEE 61 KL GVMLVGWGGNNGSTLTGGVIANRE GISWAT KDNIQQANYFGSLTQASAIRVGSFQGEE 61 KL GVMLVWGGNNGSTLTGGVIANRE GISWAT KDKIQQANYFGSITQASA IRVGSFQGEE 61 KLGVMLVGWGGNNGSTLTGGVIANRE GISWAT KDKIQQANYFGSLTQASA IRVGSYQGEE
P. sativum
M. truncatula
C. arietinum
G. $\max$
P. vulgaris

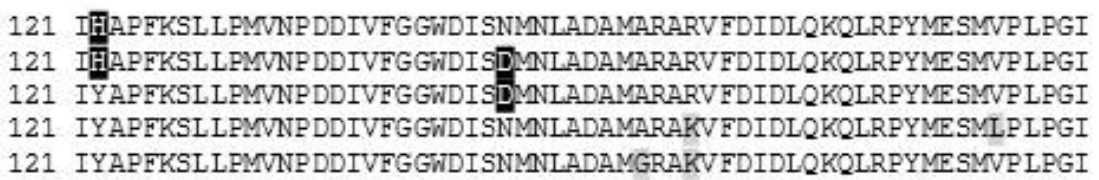

P. sativum

M. truncatula

c. arietinum

G. $\max$

P. vulgaris

P. sativum

M. truncatula

C. arietinum

G. $\max$

P. vulgaris

P. sativum

M. truncatula

C. arietinum

G. $\max$

P. vulgaris

P. sativum

M. truncatula

C. arietinum

G. $\max$

P. vulgaris
181 YDPDFIAANQGERANNIKGTKREQINQI IKD IRE FKE ANKVDRVVVLWT ANTERY SNLV 181 YD PDFIAANQGERANNVIKGTKREQI NQI IKD IKE FKE ANKVDRVVVLWT ANTERY SNLV 181 YD PDFIAANQGDRANNVIKG TKREQI NQI IKD IKE FKE ANKVDRVVVLWT ANTERY SNLV

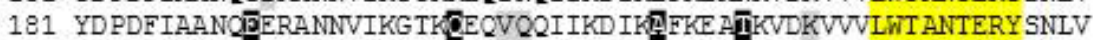

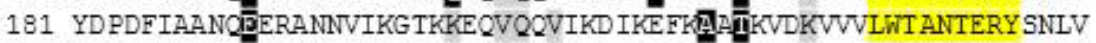

241 VGLNDTIENLFAAVDRNESE ISPSTL FGIACVMENVPF INGSPQNT FVPGLIDLAI KNNT 241 VGLNDTME NLFAAVDRNESE ISPSTL FAIACVMENVPF INGSPQNTFVPGLIDLAI KNNC 241 VGLNDTME NLFAAVDRNESE ISPSTL FAIACVTENVPF INGSPQNT FVPGLIDLAI KRNT 241 VGLNDTME NITAAVDRNEDE ISPSTL YAIACVMENVPF INGSPQNT FVPGLIDLAIRRNT

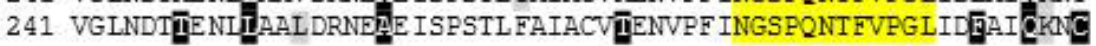

301 LIGGDDFKSGQTKMKSVLVD FLVGAG IKPTSIVSYNHL GNNDGMNLSAPQTFRSKE ISKS 301 LIGGDDFKSGQTKMKSVLVD FLVGAG IKPTSIVSYNHL GNNDGMNLSAPQTFRSKE ISKS 301 LIGGDDFKSGQTKMKSVLVD FLVGAG IKPTSIVSYNHL GNNDGMNLSAPQTFRSKE ISKS 301 LI GGDDFKSGQTKMKSVLVD FLVGAG IKPTSIVSYNHL GNNDGMNLSAPQT FRSKE ISKS 301 LI GGDDFKSGQTKMKSVLVD FLVGAG IKPTSIVSYNHL GNNDGMNLSAPQTFRSKE ISKS

361 NVVDDMVNSNAILYAPGEHP DHVVVI KYVPYVGDSKRAMDEYTSEI FMGGKNTIVLHNTC 361 NVVDDMVNSNAILYAPGEHP DHVVVI KYVPYVGDSKRAMDEYTSEIFVGGKNTIVL HNTC 361 NVVDDMVN SNGILYAPGEHP DHVVVI KYVPYVGDSKRAMDEYTSEI FMGGKSTIVL HNTC 361 NVVDDMVNSNAILYĒPGEHP DHVVVI KYVPYVGDSKRAMDEYTSEIFMGGKS̈TIVL HNTC 361 NVVDDMVNSNAILYGPGEHPDHVVVI KYVPYVADSKRAMDEYTSEIFYGGKNTIVL HNTC
P. sativum
M. truncatula
C. arietinum
G. $\max$
P. vulgaris
P. sativum
M. truncatula
c. arietinum
G. $\max$
P. vulgaris

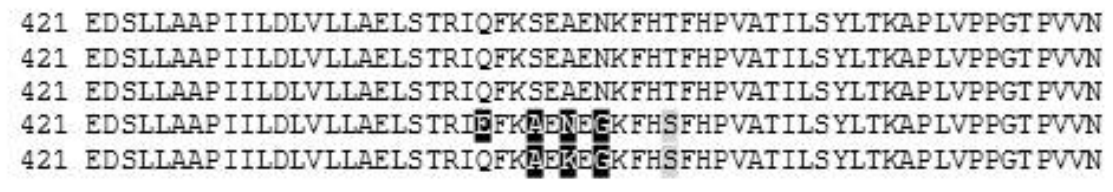

481 ALSKQRAMLENIMRACVGLA PENNMI LEYK

481 ALSKQRAMLENIMRACVGLA PENMMI LEYK

481 ALSKQRAMLENIMRACVGLA PENMMI LEYK

481 ALSKQRAMLENIMRACVGLA PENNMI LEYK

481 ALSKQRAMLENIMRACVGLAPENMI LEYK

Figure 4. Amino acid sequence alignment of PsMIPS from Pisum sativum CDC Bronco with other plant myo-inositol phosphate synthase (MIPS) sequences. Conserved domains are highlighted in yellow. Sequences were obtained from GenBank for Medicago truncatula, XP_003601987.1; Cicer arietinum, NP_001266035.1; Glycine max, ABC55420.1; and Phaseolus vulgaris, XP_007159720.1. Conserved and unconserved substitutions are indicated in black and grey boxes, respectively. 
The PsMIPS protein sequence of CDC Bronco was used in a phylogenetic analysis with 14 other MIPS sequences obtained from the National Center for Biotechnology Information (NCBI) database for a variety of plants. The phylogenetic tree presented in Figure 5 shows the evolutionary divergence among the plant MIPS sequences analyzed. The analysis confirmed PsMIPS protein sequences of CDC Bronco and the two lpa genotypes, 1-150-81 and 1-2347-144, to be 100\% identical and they clustered together. The monocots, Z. mays, O. sativa, T. aestivum, and A. sativa clearly cluster together in one branch. Two distinct sub-branches were obtained for dicots. P. sativum, M. truncatula, and C. arietinum clustered together in a branch, while G. max and P. vulgaris formed the other branch. MIPS from R. communis, $S$. tuberosum, A. deliciosa, and G. hirsutum were placed in a separate branch. A. thaliana and B. napus aligned together as an individual branch.

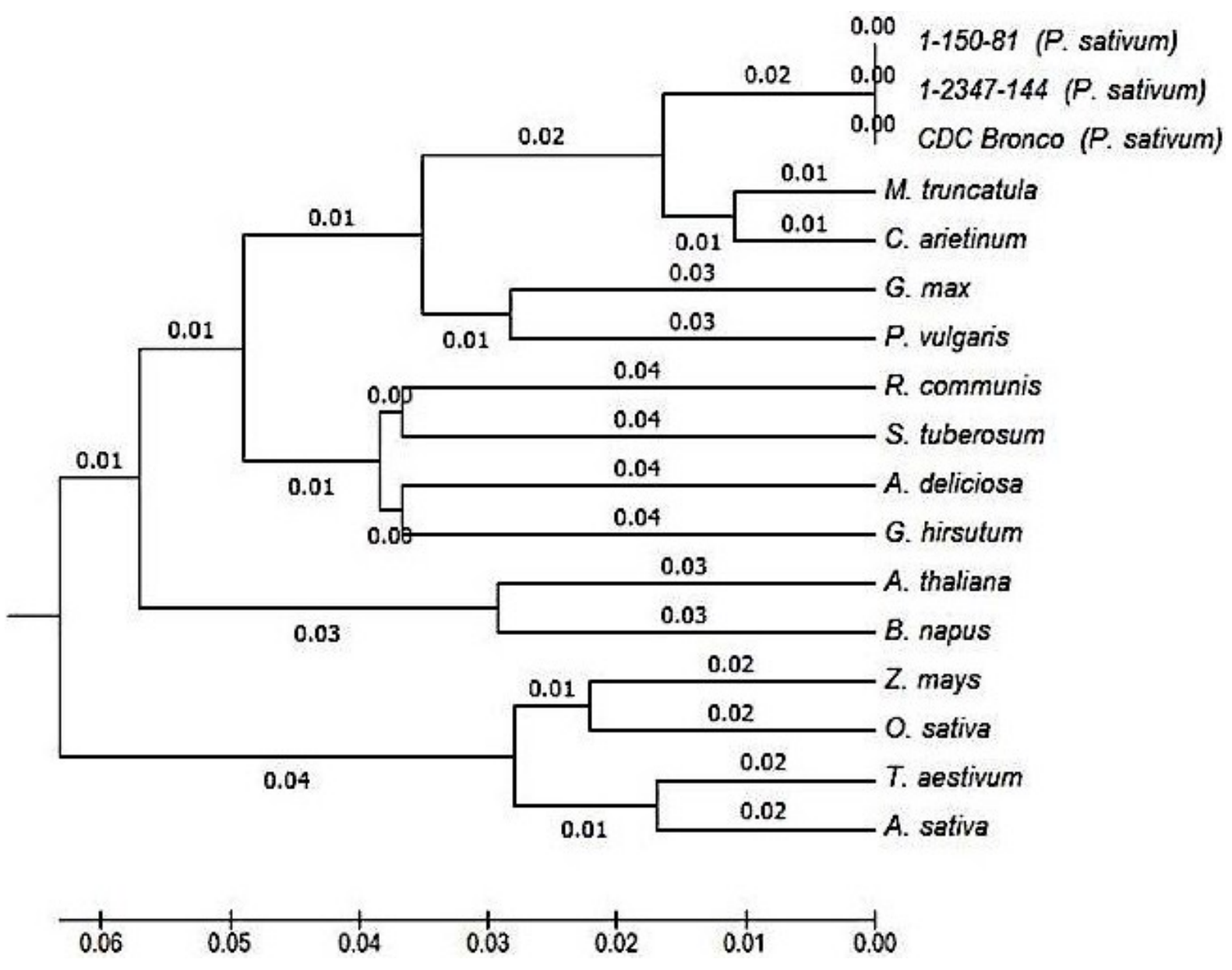

Figure 5. Phylogenetic analysis of MIPS proteins from different plant species including Pisum sativum L., CDC Bronco, and low-phytate genotypes 1-150-81 and 1-2347-144. GenBank Accession Numbers include: Medicago truncatula, XP_003601987.1; Glycine max, ABC55420.1; Cicer arietinum, NP_001266035.1; Phaseolus vulgaris, XP_007159720.1; Ricinus communis, ACU30131.1; Zea mays, ACG33827.1; Oryza sativa, BAA25729.1; Triticum aestivum, AEQ61648.1; Arabidopsis thaliana, NP_179812.1; Actinidia deliciosa, AFV31635.1; Brassica napus ACJ65004.1; Solanum tuberosum, XP_006366474.1; Gossypium hirsutum, ACJ11714.1; Avena sativa, BAB40956.2. The scale bar represents 0.05 substitutions per amino acid site, reflected in the lengths of the branches. Bootstrap values from 1000 iteration analyses are shown in italics. 


\subsection{PsMIPS Gene Expression}

An examination of the PSMIPS transcript profile in developing seeds by sqPCR revealed decreased expression levels for CDC Bronco, 1-150-81, and 1-2347-144 at 49 DAF when compared to 14 DAF (Figure 6). In comparison to CDC Bronco, PSMIPS expression at 14 DAF was somewhat decreased in 1-150-81 and elevated in 1-2347-144, while levels were essentially the same at 49 DAF (Figure 6).

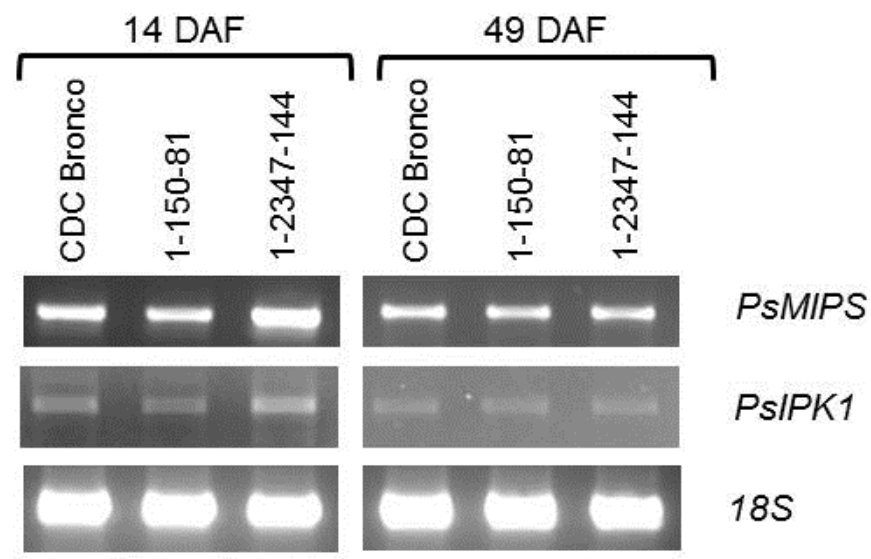

Figure 6. Transcript abundance of PsMIPS in developing seeds of pea (Pisum sativum L.) for CDC Bronco and low-phytate genotypes (1-150-81 and 1-2347-144) at 14 DAF and $49 \mathrm{DAF}$ as indicated. A fragment of $18 S$ rRNA used as a loading control is also shown. Results are representative from a minimum of three independent experiments. DAF, days after flowering.

\section{Discussion}

\subsection{Agronomic Traits in Low-Phytate Pea}

Genes, alleles, and environmental conditions affect the agronomic performance of all crops. It has been demonstrated that lpa crops can be generated without major compromises in plant performance [30]. The agronomic performance of 1-150-81 and 1-2347-144 did not significantly differ from CDC Bronco when assessed for traits such as percent emergence, plant height, lodging score, days to flowering, and days to maturity. In soybean lpa genotypes, a reduced seedling emergence was observed [31]. The difference in temperature during seed filling was proposed as the reason for the reduced emergence in the low phytate genotypes. Warkentin et al. [14] showed that 1-150-81 and 1-2347-144 were slightly different from CDC Bronco in days to flowering and days to maturity. They reported that the 1-150-81 and 1-2347-144 flowered 3 days later and matured 2 to 3 days later than CDC Bronco. This was not observed in the present study. However, the grain yield at maturity was significantly different between 1-150-81, 1-2347-144 and CDC Bronco, with 1-150-81 and 1-2347-144 showing 16\% and 17\% lower grain yields than CDC Bronco, respectively. This is similar to differences in grain yield observed by Warkentin et al. [14]. Since the phytic acid biosynthesis pathway is active in most tissues of a plant, lpa mutations can also affect the vegetative processes apart from phytate accumulation [4]. Lower seed weight might be attributed to a reduction in starch accumulation resulting from a defective inositol phosphate synthesis pathway [32]. By targeting a specific gene or its expression to appropriate seed 
tissues, low-phytate crops can be produced restoring their seed weight and yield [4]. Embryo-specific silencing of expression of an ATP binding cassette (ABC) transporter in maize produced seeds with low-phytic acid with no adverse effect on seed weight [33]. When the myo-inositol methyltransferase (IMT) gene was transferred to Brassica napus through a transgenic approach, a 19\% to 35\% reduction in phytate was achieved without affecting the seed parameters [34]. In addition, rice low-phytate mutants produced through RNAi mediated seed-specific silencing of the inositol pentakisphosphate 2-kinase (IPK1) gene, had no undesirable agronomic characters [35]. Bregitzer and Raboy [36] showed that under irrigated and non-stressful production environments, the barley lpa1-1, 1pa2-1, and lpa3-1 produced similar yields as that of the wild type barley. When the low phytate barley cultivar resulting from M640 was evaluated in distinct environments, it had a significantly higher yield when compared with commercial cultivars [37]. These studies provide evidence that low-phytate mutants can be produced in crop varieties without any compromise in agronomic traits.

\subsection{Redistribution of Phosphorus in Low-Phytate Pea during Seed Development}

A large fraction of nutrient $\mathrm{P}$ taken up by crop plants is ultimately packaged into seed phytic acid, and this single small molecule represents a major pool in the flux of $\mathrm{P}$ in the world's agricultural ecology. As phytic acid represents a significant portion of total seed $\mathrm{P}$, the accumulation of phytate and inositol phosphates has been studied in different plant species. Grain crops typically contain about $10 \mathrm{mg} \cdot \mathrm{g}^{-1}$ phytic acid on a seed dry weight basis, representing about 65 to $85 \%$ of seed total P $[38,39]$. Total $\mathrm{P}$ concentration typically ranges from 3 to $4 \mathrm{mg} \cdot \mathrm{g}^{-1}$ in seed produced by grain crops, with phytic acid-P $(\mathrm{P} P \mathrm{~A} A)$ ranging from 2 to $3 \mathrm{mg} \cdot \mathrm{g}^{-1}$.

In this study, the concentration of $\mathrm{IP}_{6}$ and its lower isomeric forms $\left(\mathrm{IP}_{1}, \mathrm{IP}_{3}, \mathrm{IP}_{4}\right.$ and $\left.\mathrm{IP}_{5}\right)$ were analyzed separately in seed coats and cotyledons. There was no traceable amount of inositol phosphates in seed coats. The accumulation of IP 6 was continuous and linear throughout the stages of seed development and the highest concentration was observed at $49 \mathrm{DAF}$. The other myo-inositol polyphosphates $\left(\mathrm{IP}_{3}, \mathrm{IP}_{4}\right.$ and $\mathrm{IP}_{5}$ ) and $\mathrm{IP}_{1}$ were not present in traceable amounts. In soybean wild-types [40] and maize lpal-1, lpa2-1 and wild types [10], phytate was reported to accumulate gradually during seed development. $\mathrm{P}_{\mathrm{i}}$ concentration decreased during seed development in wild type, and total P levels remained relatively consistent. The maize lpa genotypes had little to no increase in phytate, and $\mathrm{P}_{\mathrm{i}}$ concentration was high and did not decrease during development. In barley, the final levels and partitions of $\mathrm{P}$ forms are well documented, however it is unclear at what point during seed development the expression of the lpa genotype is initiated and how seed $\mathrm{P}$ accumulates in developing seeds of different barley lpa genotypes [41]. Israel et al. [42] compared the changes in seed phytic acid concentrations as well as myo-inositol phosphates during seed development between lpa and normal phytate genotypes and found that $\mathrm{IP}_{3}$ content was relatively low in all genotypes and decreased during seed maturation. The other inositol phosphates such as $\mathrm{IP}_{4}$ and $\mathrm{IP}_{5}$ were not detected. Larson et al. [43] reported that a reduction in seed phytate results in a molar equivalent increase in $\mathrm{P}_{\mathrm{i}}$ in rice. In the present study, $\mathrm{P}_{\mathrm{i}}$ concentration in the two lpa genotypes was $72 \%$ to $84 \%$ higher than in CDC Bronco. Between the two lpa genotypes, 1-2347-144 had 11\% more $\mathrm{P}_{\mathrm{i}}$ than 1-150-81. Throughout the developing stages, $\mathrm{P}_{\mathrm{i}}$ concentration was higher at $14 \mathrm{DAF}$ and decreased as $\mathrm{IP}_{6}$ accumulation began. Thus, there appears to be an inverse

relationship between $\mathrm{P}_{\mathrm{i}}$ accumulation and phytic acid content. The total $\mathrm{P}$ concentration showed no 
significant difference between CDC Bronco, 1-150-81, and 1-2347-144 except at 21 DAF where CDC Bronco and 1-2347-144 had 8\% and 6\% more total $\mathrm{P}$, respectively, than 1-150-81. This demonstrates that despite variations in $\mathrm{IP}_{6}$ and $\mathrm{P}_{\mathrm{i}}$ levels, the total $\mathrm{P}$ content in the seeds is unaffected. The significantly higher Pi concentration when compared to CDC Bronco in lpa pea genotypes shows that the lpa genotypes tend to balance the total $\mathrm{P}$ levels within the seed to provide adequate amounts of $\mathrm{P}$ required for P-related mechanisms in the seed. Unlike the normal genetic and environmental effects that result in quantitative variation in seed total $\mathrm{P}$, lpa mutants show large effects on the partitioning of $\mathrm{P}$ into phytic acid $\mathrm{P}$ and $\mathrm{P}_{\mathrm{i}}$.

\subsection{Myo-Inositol-3-Phosphate Synthase (MIPS) Is Identical in Normal and Low-Phytate} Pea Genotypes

Genetic mapping and comparison of the position of lpa mutation with MIPS loci in rice have been reported by Larson et al. [43]. They mapped the rice MIPS gene on to a locus on chromosome 3, which was orthologous to the MIPS gene near maize lpal on chromosome 1S. Previously, MIPS gene expression proximal to the site of phytic acid synthesis during grain development in rice was demonstrated by Yoshida et al. [44]. Hitz et al. [26] confirmed a mutation in MIPS responsible for LR33 lpa mutation in soybean. Furthermore, higher MIPS expression was found in wheat genotypes with high phytic acid levels compared to an lpa genotype [45]. These findings have contributed to the interest in MIPS as a target for manipulation to produce low phytate crops. Ipa genotypes of Arabidopsis, potato, rice, soybean, and canola have been generated by down-regulation (antisense, RNA interference, or cosuppression) or mutations in MIPS gene [24,46-48]. Bioinformatic and sqPCR analyses in this study provide evidence that the isolated MIPS gene was specific to phytic acid accumulation in seeds of both the low and normal phytate genotypes. However, the current study has shown that there are no mutations in the PSMIPS nucleotide sequences in the lpa pea genotypes.

Our sqPCR analysis revealed that at 14 DAF the expression of PSMIPS was higher in 1-2347-144 than CDC Bronco and 1-150-81. However, at 49 DAF, its expression was similar in both lpa genotypes and CDC Bronco. This further confirms that the reduction in phytate levels caused by lpa mutations is not directly controlled by MIPS expression. Moreover, mutations affecting MIPS are often associated with lower seed yield, seed viability, increased susceptibility to pathogens, and undesired morphology $[46,47,49]$ and, although the seed weight in lpa pea genotypes was lower compared to their normal progenitor, the phytate reduction was not detrimental. Therefore, we suggest that the mutation could have occurred in other genes involved in the early stages of the phytate biosynthetic pathway, such as myo-inositol kinases and 2-phosphoglycerate kinase. For example, Shi et al. [50] found that the maize lpa2 mutant with 30\% less phytic acid and three-fold more $\mathrm{P}_{\mathrm{i}}$ was caused by a mutation in an inositol phosphate kinase gene. Stevenson-Paulik et al. [51] generated phytate-free seeds in Arabidopsis through disruption of inositol polyphosphate kinases. To gain further insights into the mutation causing the low phytate phenotype in pea, it is necessary to analyze the other enzymes related to phytate biosynthesis. The other possibility is that the mutation could have affected the transport of phytic acid to the vacuole [25]. Mutations in phytic acid ATP-binding cassette transporter reduce the phytic acid content significantly and may also result in absence of lower inositol phosphates [25]. This may possibly explain the fact that there was no lower inositol phosphates detected in this study. 


\section{Experimental Section}

\subsection{Plant Material and Growth Conditions}

Seeds of two lpa field pea (Pisum sativum L.) genotypes (1-150-81 and 1-2347-144; [14], and their progenitor, a normal phytate genotype (CDC Bronco) [52] were obtained from the Crop Development Centre at the University of Saskatchewan. A four-replicate randomized complete block field trial was conducted at two locations in Saskatchewan (Rosthern and Saskatoon) in 2010 and 2011. Field trials were managed using standard techniques for field pea production in Saskatchewan that have been described earlier [14]. Seeding in both years was conducted between May 14 and May 18. Flowers were tagged at the time of flowering and developing seeds were taken 7 days after flowering (DAF) and every $7 \mathrm{~d}$ thereafter until maturity on day 49. Pea pods were collected in Ziploc ${ }^{\circledR}$ bags, transported to the laboratory on ice packs and stored at $-80{ }^{\circ} \mathrm{C}$ until use. Final harvesting in both years was conducted between August 27 and September 17. Field plots were evaluated for several phenotypic parameters during the growing season in each year. These included percent emergence, days to flower, days to maturity, plant height, mycosphaerella blight score, lodging, grain yield and 1000 seed weight. The details of these determinations have been described previously [14].

For molecular studies, seeds of the aforementioned genotypes were sown in $15 \mathrm{~cm}$ plastic pots filled with Sunshine ${ }^{\circledR}$ Mix \#3/LG3 (Sun Gro Horticulture Canada Ltd., Seba Beach, AB, Canada) and plants grown in a controlled environment chamber (PGR15; Conviron, Winnipeg, MB, Canada) set at 23/18 ${ }^{\circ} \mathrm{C}$ (day/night) temperatures with $16 \mathrm{~h}$ day length. The chamber was illuminated with fluorescent lights (T5/HO/835; Sylvania) to provide a photosynthetic photon flux density (PPFD) of $400 \mu \mathrm{mol}$ photons $\mathrm{m}^{-2} \cdot \mathrm{s}^{-1}$. Plants were regularly provided with a water-soluble fertilizer (Plant-Prod ${ }^{\circledR} 20-20-20$ Classic; Plant Products Co. Ltd., Brampton, ON, USA) and irrigated with $\mathrm{ddH}_{2} \mathrm{O}$ as required. The experiment consisted of four biological replicates for each genotype. Each replicate consisted of four pots with two plants per pot. Developing seeds were collected at the same time intervals as described above and frozen in liquid nitrogen.

\subsection{Extraction and Detection of Inositol Phosphates}

Seed coats were dissected from the cotyledons and each component was freeze dried (FreeZone 6 Liter Console Freeze Dry System; Labconco, Kansas City, MO, USA) and stored at $-80{ }^{\circ} \mathrm{C}$ until use. Seed coat or cotyledons were ground using glass beads to a $0.5 \mathrm{~mm}$ diameter using a custom designed mill powered with an inverter drive (SM-PLUS Sub-Micro; Leeson Corporation, Grafton, WI, USA). Samples were stored at $-20{ }^{\circ} \mathrm{C}$ until extraction. Inositol phosphates were extracted from the samples $(100 \mathrm{mg})$ using the extraction method described in [53] with minor modifications [54]. Extracts were filtered using $0.45 \mu \mathrm{m}$ Acrodisc ${ }^{\circledR}$ syringe filters ( $25 \mathrm{~mm}$; Pall Corporation, Port Washington, NY, USA) and used immediately for high-performance liquid chromatography (HPLC) analysis.

Detection of inositol phosphates was performed using anion-exchange high performance liquid chromatography (HPLC) on a Dionex ICS 3000 BioLC $^{\circledR}$ system (Dionex, Sunnyvale, CA, USA) and a protocol similar to those reported previously [53,54]. The system consisted of an AS50 Autosampler with a $100 \mu \mathrm{L}$ injection loop, an AS50 Thermal Compartment (set at $30{ }^{\circ} \mathrm{C}$ ), a GP50 Gradient Pump and an ED50 Electrochemical Detector coupled with an Anion Self-Regenerating Suppressor (ASRS 300, 4-mm) 
running in external water mode and a current of $297 \mathrm{~mA}$. Separation was achieved using an OmniPac PAX-100 analytical anion exchange column (Dionex; $8.5 \mu \mathrm{m}, 4 \times 250 \mathrm{~mm}$ ) preceded by an OmniPac PAX-100 guard column (Dionex; $8.5 \mu \mathrm{m}, 4 \times 50 \mathrm{~mm}$ ). Inositol phosphates were separated with a multi-step gradient using water purified by a Milli-Q Water System (Millipore, Milford, MA, USA) to a resistance of $\geq 18 \mathrm{~m} \Omega$. Mobile phases included were water (A), $200 \mathrm{mM} \mathrm{NaOH}$ (B), and water/isopropanol (50:50, v/v) (C). The total run time was $80 \mathrm{~min}$, which included an equilibration to starting conditions. A column flow rate of $1.0 \mathrm{~mL} \cdot \mathrm{min}^{-1}$ was maintained for the mobile phase flow, with a linear gradient profile consisting of solvent $\mathrm{A}$ with the following proportions $(\mathrm{v} / \mathrm{v})$ of solvent $\mathrm{B}$ or $\mathrm{C}$ : 0-13 min, $\mathrm{B}=6 \%$ and $\mathrm{C}=12 \% ; 13-30 \mathrm{~min}, \mathrm{~B}=30 \%$ and $\mathrm{C}=2 \% ; 30-43 \mathrm{~min}, \mathrm{~B}=56 \%$ and $\mathrm{C}=2 \%$; $43-55 \mathrm{~min}, \mathrm{~B}=56 \%$ and $\mathrm{C}=2 \% ; 55-65 \mathrm{~min}, \mathrm{~B}=61 \%$ and $\mathrm{C}=8 \% ; 65-80 \mathrm{~min}, \mathrm{~B}=6 \%$ and $\mathrm{C}=2 \%$. Chromeleon software (Dionex) was used to plot chromatograms and analyze the data. A standard solution was prepared for each of IP 6 and its lower isomeric forms $\mathrm{IP}_{1}, \mathrm{IP}_{3}, \mathrm{IP}_{4}, \mathrm{IP}_{5}$ (\#P8810, \#I1267, \#I7012, \#I5514, \#I9261, respectively; all from Sigma-Aldrich, St. Louis, MO, USA). This was used to establish retention times for these compounds, which were subsequently used to determine peak identity in the samples. Inositol phosphate quantification was afforded using external standard curves with $R^{2}$ values of 0.99 or greater for each compound. Concentrations for each of the inositol phosphate standards ranged from 1.25 to $25.0 \mathrm{ppm}$. Phytic acid phosphorus ( $\left.\mathrm{P}_{\mathrm{PhA}}\right)$ was calculated as the number of moles of phytic acid/3.56 as described by [54], assuming all $\mathrm{P}_{\mathrm{PhA}}$ comes from IP6.

\subsection{Analysis of Phosphorus Levels}

Inorganic phosphorus $\left(\mathrm{P}_{\mathrm{i}}\right)$ levels were determined as described by [14]. Ground cotyledon samples (50 mg) were extracted overnight at $4{ }^{\circ} \mathrm{C}$ in $1 \mathrm{~mL}$ of $0.4 \mathrm{M} \mathrm{HCl}$ followed by vigorous mixing. A $10 \mu \mathrm{L}$ aliquot of the extract was aliquoted into a microtiter plate with $90 \mu \mathrm{L}$ of $\mathrm{ddH}_{2} \mathrm{O}$ and $100 \mu \mathrm{L}$ of freshly prepared Chen's reagent. Chen's reagent contains $6 \mathrm{~N} \mathrm{H}_{2} \mathrm{SO}_{4}, 2.5 \%(\mathrm{w} / \mathrm{v})\left(\mathrm{NH}_{4}\right)_{2} \mathrm{MoO}_{4}$ (ammonium molybdate), $10 \%$ (w/v) ascorbic acid, and $\mathrm{ddH}_{2} \mathrm{O}$ (1:1:1:2) [54]. The mixtures were incubated for two hours at room temperature before reading the $A 655$ with a microplate absorbance spectrophotometer (xMark ${ }^{\mathrm{TM}}$; Bio-Rad Laboratories, Hercules, CA, USA) against a water blank. Standard curves of $\mathrm{K}_{2} \mathrm{HPO}_{4}$ were constructed ranging from 10 to $50 \mathrm{ppm}$ with $R^{2}$ values of 0.95 or greater. Sample values were interpolated from these curves and expressed on a dry weight (DW) basis.

Total $\mathrm{P}$ in cotyledons was assayed by the wet ashing method [10]. Ground samples (50 mg) were incubated with $1 \mathrm{~mL}$ of concentrated $(18.4 \mathrm{M}) \mathrm{H}_{2} \mathrm{SO}_{4}$ overnight at room temperature. Two hundred microliters of $30 \%(\mathrm{v} / \mathrm{v}) \mathrm{H}_{2} \mathrm{O}_{2}$ were added and the samples were incubated in a heating block between 220 and $250{ }^{\circ} \mathrm{C}$ for $30 \mathrm{~min}$. Samples were removed and allowed to cool at room temperature for $15 \mathrm{~min}$. This cycle was repeated until the sample became clear. The volume of the samples was adjusted to $6.25 \mathrm{~mL}$ with $\mathrm{ddH}_{2} \mathrm{O}$ and total extractable $\mathrm{P}$ was determined spectrophotometrically using the method of [55] as described above.

\subsection{Reverse Transcriptase-Polymerase Chain Reaction (RT-PCR) and Sequencing}

Total RNA was isolated from $75 \mathrm{mg}$ of seed from 14 DAF and 49 DAF samples with an RNeasy ${ }^{\circledR}$ Plant Mini Kit (Qiagen, Valencia, CA, USA) using the RLC buffer according to the manufacturer's instructions. The RNA was eluted in $30 \mu \mathrm{L}$ of RNase-free water. The samples were quantified $\left(A_{260}\right)$ and 
purity $\left(A_{260}: A_{280}\right)$ determined using a Nanodrop 8000 spectrophotometer (Thermo Fisher Scientific, Ottawa, ON, USA). Gel electrophoresis on denaturing 1.2\% (w/v) agarose gels containing formaldehyde was used to examine the quality of the RNA. This was assessed by the sharpness of the rRNA bands and 2:1 ratio of $28 \mathrm{~S}$ rRNA to $18 \mathrm{~S}$ rRNA. Gels were run in $1 \times 3-(\mathrm{N}$-morpholino) propanesulfonic acid (MOPS) buffer and stained with ethidium bromide (Sambrook and Russell 2001). The isolated RNA was stored at $-80{ }^{\circ} \mathrm{C}$ until further use. cDNA was synthesized from $100 \mathrm{ng}$ of total RNA using the QuantiTect ${ }^{\circledR}$ Reverse Transcription Kit (Qiagen, Valencia, CA, USA) as described by the supplier.

The annotated Cool Season Food Legume Genome Database (http://www.coolseasonfoodlegume.org/) [56] was searched for MIPS and a contig in Pisum sativum identified (Pisum_sativum_v2_Contig5216) which contained the entire coding region of the PSMIPS gene, confirmed by translation and alignment with alfalfa (Medicago sativa) MIPS (GenBank Accession Number EF408869.1). Gene specific primers for PSMIPS were designed from this contig using the Primer-BLAST tool (http://www.ncbi.nlm.nih.gov/tools/primer-blast/) [57] at the National Center for Biotechnology Information (NCBI) to amplify a 1602-bp fragment encompassing the 1530-bp open reading frame (ORF). cDNA was amplified by polymerase chain reaction (PCR) using an iCycler (Bio-Rad Laboratories) and a thermostable DNA polymerase (Q5 High-Fidelity; New England Biolabs, Whitby, ON, USA). Forward (5'-ATGTTCATAGAGAGTTTCAAGGTTGAGAGT-3') and reverse (5'-GCTTGTGTTGGATTGGCTCCAGA-3') primers were used at a final concentration of $0.5 \mu \mathrm{M}$ each, and $2 \mu \mathrm{L}$ of the cDNA reaction was used as a template in the $25 \mu \mathrm{L}$ PCR reaction. The following cycling conditions were used: cDNA denaturation at $98^{\circ} \mathrm{C}$ for $30 \mathrm{~s}$, then 35 cycles of denaturation at $98^{\circ} \mathrm{C}$ for $10 \mathrm{~s}$, annealing at $55^{\circ} \mathrm{C}$ for $30 \mathrm{~s}$, extension at $72{ }^{\circ} \mathrm{C}$ for $1 \mathrm{~min}$, followed by a final extension at $72{ }^{\circ} \mathrm{C}$ for $2 \mathrm{~min}$. PCR products were visualized on a $1.2 \%(\mathrm{w} / \mathrm{v})$ agarose gel run in $1 \times$ Tris-Acetate-EDTA (TAE) and stained with ethidium bromide [58]. The PCR product corresponding to 1602-bp was excised from the gel and extracted using the QIAPrep Gel Extraction Kit (Qiagen) according to the manufacturer's instructions. Extractions from multiple PCR reactions were pooled and directly sequenced using the BigDye $^{\circledR}$ Terminator v3.1 Cycle Sequencing Kit (Applied Biosystems, Foster City, CA, USA) at the National Research Council of Canada (NRC; Saskatoon, SK, Canada).

\subsection{Semi-Quantitative Reverse Transcriptase-Polymerase Chain Reaction (sqRT-PCR)}

Transcript levels of PSMIPS were examined using sqRT-PCR and performed using the Verso 1-Step RT-PCR ReddyMix ${ }^{\mathrm{TM}}$ Kit (Thermo Fisher Scientific, Ottawa, ON, USA) as recommended by the supplier. Targets were amplified using specific primers to PSMIPS or pea $18 S$ small subunit nuclear rRNA. Primers (forward primer, 5'-CATTGGAGGGCAAGTCTGGT-3'; reverse primer, 5'-CCAGCGGAGTCCTAAAAGCA-3') for pea $18 S$ (GenBank accession number U43011.1) generated a 510-bp amplicon that was used as a reference gene [59]. RNA was isolated as described above and $100 \mathrm{ng}$ was used as template in the $25 \mu \mathrm{L}$ reactions. The following cycling conditions were used: cDNA synthesis at $50{ }^{\circ} \mathrm{C}$ for $15 \mathrm{~min}$, enzyme inactivation at $95{ }^{\circ} \mathrm{C}$ for $2 \mathrm{~min}$, then 40 cycles of denaturation at $95{ }^{\circ} \mathrm{C}$ for $20 \mathrm{~s}$, annealing at $55^{\circ} \mathrm{C}$ for $30 \mathrm{~s}$, extension at $72{ }^{\circ} \mathrm{C}$ for $1 \mathrm{~min}$, followed by a final extension at $72{ }^{\circ} \mathrm{C}$ for $5 \mathrm{~min}$. PCR products were visualized on a $1.2 \%(\mathrm{w} / \mathrm{v})$ agarose gel run in $1 \times \mathrm{TAE}$ and stained with ethidium bromide [58]. 


\subsection{Sequence Analysis}

Sequencing results were assembled using the BioEdit sequence alignment editor (v7.2.5, http://www.mbio.ncsu.edu/BioEdit/bioedit.html) [60]. Deduced amino acid sequences were obtained using the Translate Tool on the ExPASy SIB Bioinformatics Resource Portal (http://web.expasy.org/ translate/). MIPS nucleotide and protein sequences were obtained from GenBank (www.ncbi.nih.nlm.gov) at NCBI and aligned using BLAST (http://blast.ncbi.nlm.nih.gov/Blast.cgi) [61] and CLUSTALW2.1 at the European Bioinformatics Institute (http://www.ebi.ac.uk/Tools/msa/clustalw2/) [62]. Domain analysis of MIPS amino acid sequences was performed using InterProScan (http://www.ebi.ac.uk/ interpro/interproscan.html) [63]. Phylogeny analysis was performed using the MEGA6 program (http://www.megasoftware.net/) [64] and available amino acid sequences (NCBI protein sequence database; http://www.ncbi.nlm.nih.gov/nuccore).

\subsection{Statistics}

All statistical analyses were performed using SAS ${ }^{\circledR} 9.3$ (SAS Institute Inc., Cary, NC, USA). The experimental data presented are mean values from two locations over two years. Levene's test was conducted to analyze the homogeneity of variance and the results represent means \pm standard error (SE), based on four replications. Significant differences were determined by a one-way analysis of variance (ANOVA), $p<0.05$. Differences among the means were analyzed by a least significant difference (LSD) post-hoc test at $p<0.05$.

\section{Conclusions}

In conclusion, this study describes the accumulation patterns of phosphorus compounds in developing seeds of two low-phytate pea genotypes in comparison to their progenitor CDC Bronco. Based on the presented evidence, the possibility of a MIPS mutation being responsible for the low phytate trait in these pea genotypes is excluded. Interestingly, no accumulation of lower inositol polyphosphates was observed. Once the causative mutation is identified in these low-phytate pea genotypes, it can then be mapped and used in marker-assisted breeding to select low-phytate genotypes.

\section{Acknowledgments}

Financial support for this research was provided by the Saskatchewan Pulse Growers, the Natural Science and Engineering Research Council (NSERC) of Canada and the University of Saskatchewan. Work in GRG's lab was supported by a NSERC Discovery grant. The technical support of the pulse crop breeding crew at the Crop Development Centre is gratefully acknowledged.

\section{Author Contributions}

ASKS performed the fieldwork, phenotypic assays, molecular biology experiments, and drafted the manuscript. CB co-ordinated the HPLC assays and contributed to data interpretation. GCA co-ordinated the calorimetric assays. FG co-conceptualized the study and assisted with the data interpretation. GRG co-ordinated the molecular biology experiments, interpreted sequence data, and 
helped to draft the manuscript. TDW conceptualized and co-ordinated the study. All authors read and approved the final manuscript.

\section{Appendix}

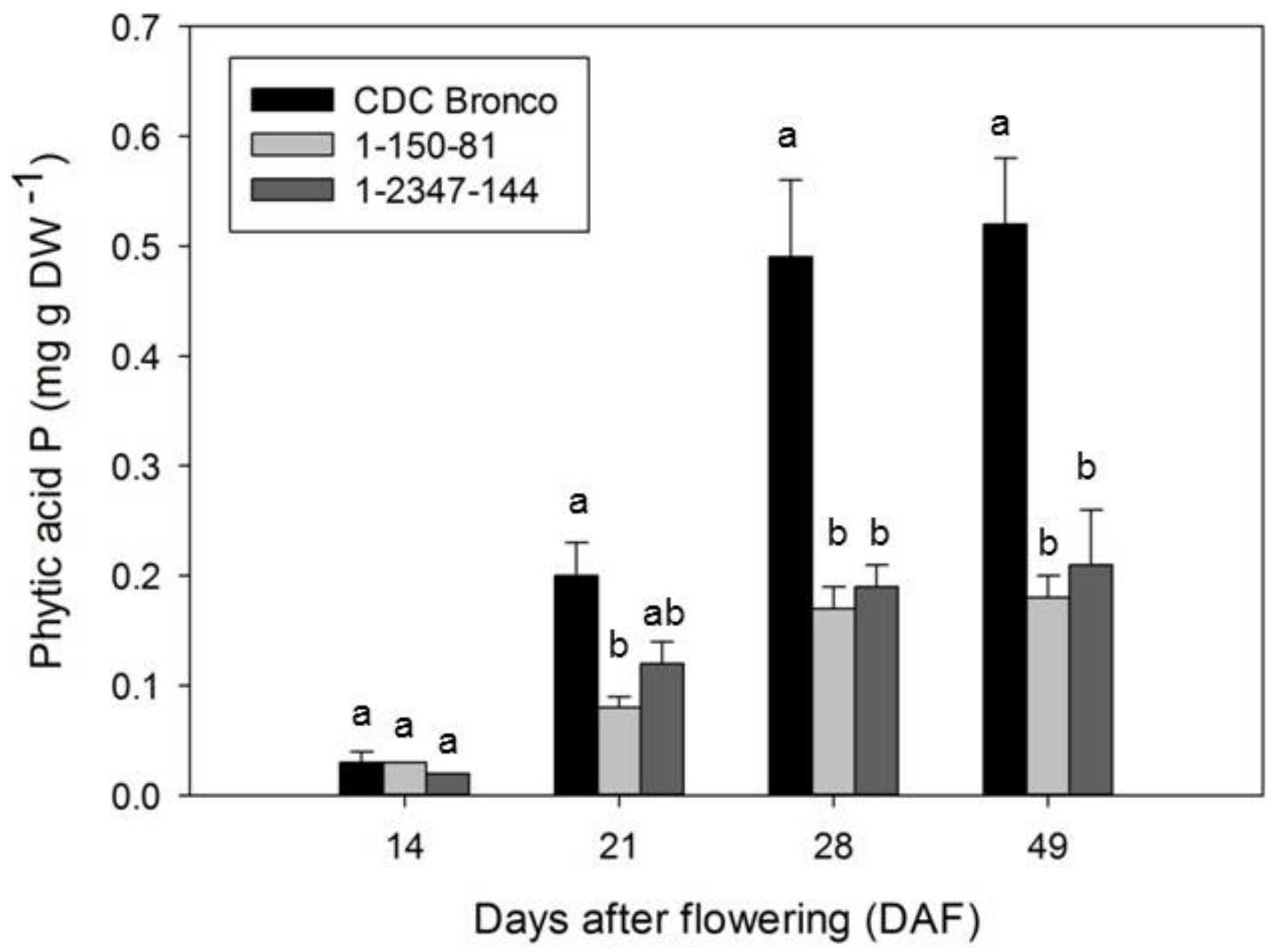

Figure A1. Content of phytic acid P (PPhA) in developing seeds of pea (Pisum sativum L.) for CDC Bronco (black bars) and low-phytate genotypes 1-150-81 (light grey bars) and 1-2347-144 (dark grey bars). Values represent means $\pm \mathrm{SE}, n=4$. Different letters associated with bars within each DAF are significantly different at $p<0.05$. DAF, days after flowering; DW, dry weight; P, phosphorus PphA, phytic acid phosphorus. 


CDC Bronco
$1-150-81$
$1-2347-144$
M. truncatula
G. max

CDC Bronco

$1-150-81$

1-2347-144

M. truncatula

G. $\max$

CDC Bronco

1-150-81

$1-2347-144$

M. truncatula

G. $\max$

CDC Bronco

$1-150-81$

$1-2347-144$

M. truncatula

G. $\max$

CDC Bronco

$1-150-81$

$1-2347-144$

M. truncatula

G. $\max$

CDC Bronco

$1-150-81$

$1-2347-144$

M. truncatula

G. $\max$

CDC Bronco

$1-150-81$

$1-2347-144$

M. truncatula

G. $\max$

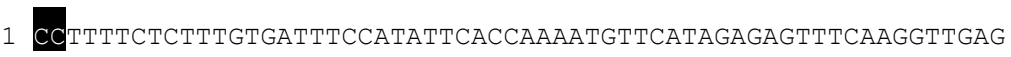

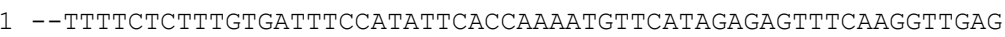

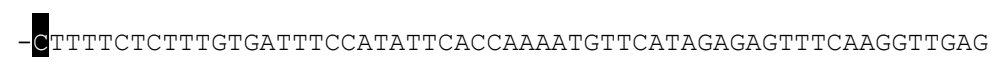
ATGTTTATCGAGAATTTCAAAGTCGAG

-GTGAAAAATAATGTCATCGAGAATtTAAAGGTGAG
61 AGTCCTAACGTGAAGTACACAGACACAGAGATTCAGTCTGTGTACAGTTACGAAACAACT
59 AGTCCTAACGTGAAGTACACAGACACAGAGATTCAGTCTGTGTACAGTTACGAAACAACT
60 AgtCCTAACGTGAAGTACACAGACACAGAGATTCAGTCTGTGTACAGTTACGAAACAACT 28 AGTCCCAATGTTAAGTACACTGAAACTGAGATTCAATCCGTTTACAATTACGAAACCACT
38 TGTCCTAATgTGAAGTACACCGAGACTGAGATTCAGTCCGTGTACAACTACGAAACCACC

121 GAACTTGTTCATCAGAACAGAAATGACACTTATCAATGGATTGTTAACCCTAAAACTGTG
119 GAACTTGTTCATCAGAACAGAAATGACACTTATCAATGGATTGTTAACCCTAAAACTGTG
120 GAACTTGTTCATCAGAACAGAAATGACACTTATCAATGGATTGTTAACCCTAAAACTGTG
88 GAACTTGTTCATGAAAATCGTAATGGCACTTATCAGTGGATTGTTAAACCTAAAACTGTT
98 GAACTTGTTCACGAGAACAGGAATGGCACCTATCAGTGGATTGTCAAACCCAAATCTGTC

181 AAATATGAATTTAAAACCGAAACTCATGTTCCTAAATTGGGGGTAATGCTTGTGGGATGG 179 AAATATGAATTTAAAACCGAAACTCATGTTCCTAAATTGGGGGTAATGCTTGTGGGATGG 180 AAATATGAATTTAAAACCGAAACTCATGTTCCTAAATTGGGGGTAATGCTTGTGGGATGG 148 AAATATGAATTTAAAACCGATATTCATGTCCCTAAATTGGGGGTAATGCTTGTGGGATGG 158 AAATACGAATTTAAAACCAACATCCATGTTCCTAAATTAGGGGTAATGCTTGTGGGTTGG

241 GGTGGAAACAACGGTTCAACCCTTACCGGTGGTGTTATTGCTAATCGAGAGGGTATTTCA 239 GGTGGAAACAACGGTTCAACCCTTACCGGTGGTGTTATTGCTAATCGAGAGGGTATTTCA 240 GGTGGAAACAACGGTTCAACCCTTACCGGTGGTGTTATTGCTAATCGAGAGGGTATTTCA 208 GGTGGAAACAACGGTTCAACCCTTACCGGTGGTGTTATTGCTAACAGAGAGGGAATTTCA 218 GGTGGAAACAACGGCTCAACCCTCACCGGTGGTGTTATTGCTAACCGAGAGGGCATTTCA 301 TGgGCAACGAAAgATAATATTCAACAAGCGAATTACTTTGGTTCTCTCACTCAAGCTTCA 299 TGGGCAACGAAAGATAATATTCAACAAGCGAATTACTTTGGTTCTCTCACTCAAGCTTCA 300 TGGGCAACGAAAGATAATATTCAACAAGCGAATTACTTTGGTTCTCTCACTCAAGCTTCA

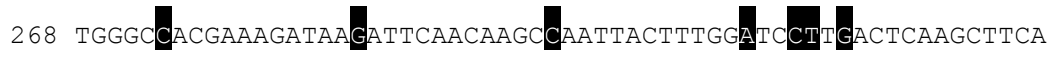

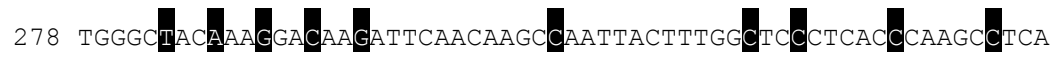
361 GCTATTCGTGTTGGATCTTTCCAAGGAGAGGAAATTCATGCTCCCTTCAAGAGCTTGTTA 359 GCTATTCGTGTTGGATCTTTCCAAGGAGAGGAAATTCATGCTCCCTTCAAGAGCTTGTTA 360 GCTATTCGTGTTGGATCTTTCCAAGGAGAGGAAATTCATGCTCCCTTCAAGAGCTTGTTA 328 GCTATTCGAGTTGGATCTTTTCAAGGAGAGGAAATTCATGCTCCTTTCAAGAGCCTCCTT 338 GCTATCCGAGTTGGGTCCTTCCAGGGAGAGGAAATCTATGCCCCATTCAAGAGCCTGCTT

Figure A2. Cont. 

CDC Bronco
421 CCAATGGTCAACCCTGATGACATTGTTTTTGGTGGATGGGATATCAGTAACATGAACCTT
$1-150-81$
$1-2347-144$
419 CCAATGGTCAACCCTGATGACATTGTTTTTGGTGGATGGGATATCAGTAACATGAACCTT
420 CCAATGGTCAACCCTGATGACATTGTTTTTGGTGGATGGGATATCAGTAACATGAACCTT
M. truncatula
G. $\max$
388 CCAATGGTCAACCCCGATGACATTGTTTTTGGTGGATGGGATATCAGTGACATGAACCTT
398 CCAATGGTTAACCCTGACGACATTGTGTTTGGGGGATGGGATATCAGCAACATGAACCTE

CDC Bronco

$1-150-81$

$1-2347-144$

M. truncatula

G. $\max$

CDC Bronco

1-150-81

$1-2347-144$

M. truncatula

G. $\max$

CDC Bronco

1-150-81

1-2347-144

M. truncatula

G. $\max$

CDC Bronco

1-150-81

1-2347-144

M. truncatula

G. $\max$

CDC Bronco

1-150-81

1-2347-144

M. truncatula

G. $\max$

CDC Bronco

1-150-81

$1-2347-144$

M. truncatula

G. $\max$
481 GCTGATGCCATGGCTAGGGCCAGGGTTTTCGACATTGATTTGCAAAAGCAATTGAGGCCT

479 GCTGATGCCATGGCTAGGGCCAGGGTTTTCGACATTGATTTGCAAAAGCAATTGAGGCCT

480 GCTGATGCCATGGCTAGGGCCAGGGTTTTCGACATTGATTTGCAAAAGCAATTGAGGCCT

448 GCTGATGCCATGGCTAGGGCCAGGGTTTTTGACATTGATTTGCAAAAGCAATTGAGGCCT 458 GCTGATGCCATGGCCAGGGCAAAGGTGTTGACATCGATTTGCAGAAGCAGTTGAGGCCT

541 TATATGGAATCCATGGTTCCACTCCCCGGCATCTATGACCCGGATTTCATTGCTGCCAAT 539 TATATGGAATCCATGGTTCCACTCCCCGGCATCTATGACCCGGATTTCATTGCTGCCAAT 540 TATATGGAATCCATGGTTCCACTCCCCGGCATCTATGACCCGGATTTCATTGCTGCCAAT 508 TATATGGAATCCATGGTTCCACTTCCCGGTATCTATGACCCGGATTTCATTGCTGCTAAT 518 TACATGGAATCCATGCTTCCACTCCCCGGAATCTATGACCCGGATTTCATTGCTGCCAAC

601 CAAGGTGAACGTGCAAATAATGTTATTAAGGGTACAAAGAGAGAACAAATTAACCAAATC 599 CAAGGTGAACGTGCAAATAATGTTATTAAGGGTACAAAGAGAGAACAAATTAACCAAATC 600 CAAGGTGAACGTGCAAATAATGTTATTAAGGGTACAAAGAGAGAACAAATTAACCAAATC 568 CAAGGAGAACGTGCGAATAACGTTATCAAGGGTACAAAGAGAGAACAAATCAACCAAATC 578 CAAGAGAGCGTGCCAACAACGTCATCAAGGGCACAAAGCAAGAGCAAGTTCAACAAATC
661 ATCAAAGACATTCGGGAATTTAAGGAAGCAAACAAAGTAGACAGGGTTGTTGTTCTCTGG 659 ATCAAAGACATTCGGGAATTAAGGAAGCAAACAAAGTAGACAGGGTTGTTGTTCTCTGG 660 ATCAAAGACATTCGGGATTTAAGGAAGCAAACAAAGTAGACAGGGTTGTTGTTCTCTGG 628 ATCAAAGACATTAAGGAATTTAAGGAAGCAAACAAAGTTGACAGGGTTGTTGTACTCTGG 638 ATCAAAGACATCAAGGCGTTTAAGGAAGCCACCAAAGTGGACAAGGTGGTTGTACTGTGG
781 CTTTTTGCTGCAGTGGACAGAAATGAGTCTGAGATTTCTCCTTCCACCCTGTTTGGCATT
779 CTTTTTGCTGCAGTGGACAGAAATGAGTCTGAGATTTCTCCTTCCACCCTGTTTGGCATT
780 CTTTTTGCTGCAGTGGACAGAAATGAGTCTGAGATTTCTCCTTCCACCCTGTTTGGCATT 748 CTTTTTGCTGCTGTGGACAGAAATGAGTCTGAGATTTCA CСTTCCACCCTGTTTGCCATT 758 CTCTTGGCTGCTGTGGACAGAAATGAGGCTGAGATTTCTCCTTCCACCTTGTATGCCATT

Figure A2. Cont. 


\begin{tabular}{|c|c|c|}
\hline CDC Bronco & 11 & GCTTGTGTTATGGAAAATGTTCCTTTCATCAATGGAAGCCCTCAGAACACTTTTGT: \\
\hline $1-150-81$ & 839 & GCTTGTGTTATGGAAAATGTTCCTTTCATCAATGGAAGCCCTCAGAACACTTTTGTTCCA \\
\hline $1-2347-144$ & 840 & GCTTGTGTTATGGAAAATGTTCCTTTCATCAATGGAAGCCCTCAGAACACTTTTGTTCCA \\
\hline M. truncatula & 808 & GCTTGTGTTATGGAGAATGTTCCTTTCATCAATGGAAGTCCTCAGAACACTTTTGTTCCA \\
\hline G. $\max$ & 818 & GCTTGTGTTATGGAAAATGTTCCTTTCATTAATGGAAGCCCTCAGAACACTTTTGTACCA \\
\hline CDC Bronco & 901 & GGGCTTATTGATCTTGCCATCAAGAACAACACCTTGATTGGTGGCGATGACTTCAAGAGT \\
\hline $1-150-81$ & 899 & GGGCTTATTGATCTTGCCATCAAGAACAACACCTTGATTGGTGGCGATGACTTCAAGAGT \\
\hline $1-2347-144$ & 900 & GGGCTTATTGATCTTGCCATCAAGAACAACACCTTGATTGGTGGCGATGACTTCAAGAGT \\
\hline M. truncatula & 868 & GGGCTTATTGATCTTGCCATCAAGAAC \\
\hline G. $\max$ & 878 & GGGCTGATTGATCTTGCCATCGCGAGGAACACTTTGATTGGTGGÄGATGACTTCAAGAGT \\
\hline CDC Bronco & 961 & GGTCAGACCAAAATGAAATCTGTTTTGGTTGATTTCCTTGTTGGAGCTGGTATCAAGCCA \\
\hline $1-150-81$ & 959 & GGTCAGACCAAAATGAAATCTGTTTTGGTTGATTTCCTTGTTGGAGCTGGTATCAAGCCA \\
\hline $1-2347-144$ & 960 & GGTCAGACCAAAATGAAATCTGTTTTGGTTGATTTCCTTGTTGGAGCTGGTATCAAGCCA \\
\hline M. truncatula & 928 & GGTCAGACCAAAATGAAATCTGTTTTGGTGGATTTCCTTGTAGGAGCTGGTATCAAGCCA \\
\hline G. $\max$ & & ETCAGACCAAAATGAAATCTGTGTTGGTTGATTTCCTTGTGGGGGCTGGTATCAAC \\
\hline
\end{tabular}

CDC Bronco 1021 ACGTCGATAgTGAgtTACAATCATCTTGGAAACAATGATGGTATGAACCTCTCAGCACCA 1-150-81 1019 ACGTCGATAGTGAGTTACAATCATCTTGGAAACAATGATGGTATGAACCTCTCAGCACCA 1-2347-144 1020 ACGTCGATAGTGAGTTACAATCATCTTGGAAACAATGATGGTATGAACCTCTCAGCACCA M. truncatula 988 ACATCAATAGTGAGTTACAATCATCTTGGAAACAATGATGGTATGAACCTCTCAGCCCCA G. max 998 ACATCTATAGTCAGTTACAACCATCTGGGAAACAATGATGGTATGAATCTITCGGCTCCA

CDC Bronco 1081 CAAACCTTCCGCTCCAAGGAAATCTCCAAGAGCAACGTTGTTGACGATATGGTCAACAgC 1-150-81 1079 CAAACCTTCCGCTCCAAGGAAATCTCCAAGAGCAACGTTGTTGACGATATGGTCAACAGC 1-2347-144 1080 CAAACCTTCCGCTCCAAGGAAATCTCCAAGAGCAACGTTGTTGACGATATGGTCAACAGC M. truncatula 1048 CAAACCTTCCGCTCCAAGGAAATTTCCAAGAGCAACGTTGTTGACGATATGGTCAACAGC G. max 1058 CAAACTTTCCGTTCCAAGGAAATCTCCAAGAGCAACGTTGTTGATGATATGGTCAACAGC

CDC Bronco 1141 AACGCTATCCTCTATGCGCCTGGTGAACATCCTGACCATGTTGTAGTCATtAagtATGtg 1-150-81 1139 AACGCTATCCTCTATGCGCCTGGTGAACATCCTGACCATGTTGTAGTCATTAAgTATgtG 1-2347-144 1140 AACGCTATCCTCTATGCGCCTGGTGAACATCCTGACCATGTTGTAGTCATTAAGTATGTG

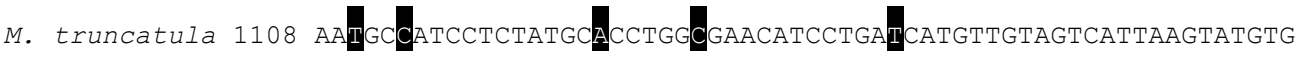
G. $\max$ 1118 AATGCCATCCTCTATGAGCCTGGTGAACATCCAGACCATGTTGTTGTTATTAAGTATGTG

CDC Bronco 1201 CCATACGTCGGAGACAgCAAGAGAGCCATGGACGAGTATACTTCGGAAATATTCATGGGT 1-150-81 1199 CCATACGTCGGAGACAGCAAGAGAGCCATGGACGAGTATACTTCGGAAATATTCATGGGT 1-2347-144 1200 CCATACGTCGGAGACAGCAAGAGAGCCATGGACGAGTATACTTCGGAAATATTCATGGGT M. truncatula 1168 CCTTATGTTGGTGACAGCAAGAGAGCTATGGATGAGTACACTTCGGAAATTTTCATGGGT G. $\max$ 1178 CCTTACGTAGGGGACAGCAAGAGAGCCATGGATGAGTACACTTCA GAGATATTCATGGGT

Figure A2. Cont. 
$\begin{array}{lrl}\text { CDC Bronco } & 1261 \text { GGAAAGAACACTATTGTGTTGCACAACACATGTGAGGATTCCCTCTTGGCTGCCCCTATT } \\ \text { 1-150-81 } & 1259 \text { GGAAAGAACACTATTGTGTTGCACAACACATGTGAGGATTCCCTCTTGGCTGCCCCTATT } \\ 1-2347-144 & 1260 \text { GGAAAGAACACTATTGTGTTGCACAACACATGTGAGGATTCCCTCTTGGCTGCCCCTATT } \\ \text { M. truncatula } 1228 \text { GGGAAGAACACTATTGTGTTGCACAACACATGTGAGGACTCCCTTTTGGCTGCTCCTATT } \\ \text { G. max } & 1238 \text { GGAAAGAGACCATTGTTTTGCACAACACATGCGAGGATTCCCTCTTAGCTGCTCCTATT }\end{array}$

CDC Bronco 1321 ATCTTGGACTTGGTTCTTCTTGCTGAGCTTAGTACTAGAATTCAGTTCAAATCTGAAGCT 1-150-81 1319 ATCTTGGACTTGGTTCTTCTTGCTGAGCTTAGTACTAGAATTCAGTTCAAATCTGAAGCT 1-2347-144 1320 ATCTTGGACTTGGTTCTTCTTGCTGAGCTTAGTACTAGAATTCAGTTCAAATCTGAAGCT

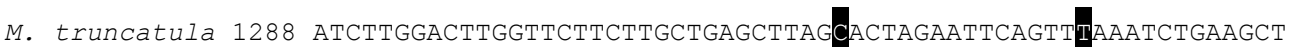
G. max 1298 ATCtTGGACTtGgtCCTTCTTGCTGAGCTCAGCACTAGAATCGAGTTIAAAGCTGAAAAT

CDC Bronco 1381 GAGAACAAGTTTCACACATTCCACCCTGTTGCTACCATCCTCAGTTATCTGACCAAGGCT 1-150-81 1379 GAGAACAAGTTTCACACATTCCACCCTGTTGCTACCATCCTCAGTTATCTGACCAAGGCT 1-2347-144 1380 GAGAACAAGTTTCACACATTCCACCCTGTTGCTACCATCCTCAGTTATCTGACCAAGGCT M. truncatula 1348 GAGAACAAGTTdCACACdTTCCACCCTGTTGCTACCATCCTCAGTTATCTGACCAAGgCT G. max 1358 GAGGGAAAMTTCACTCATTCCACCCAGTTGCTACCATCCTCAGCTACCTCACCAAGGCT

CDC Bronco 1441 CCTCTGGTTCCACCAGGTACACCAGTGGTGAATGCATTGTCCAAGCAGCGAGCGATGCTG 1-150-81 1439 CCTCTGGTTCCACCAGGTACACCAGTGGTGAATGCATTGTCCAAGCAGCGAGCGATGCTG 1-2347-144 1440 CCTCTGGTTCCACCAGGTACACCAGTGGTGAATGCATTGTCCAAGCAGCGAGCGATGCTG M. truncatula 1408 CCTCTGGTTCCACCGGGCACACCAGTGGTGAATGCATTGTCAAAGCAGCGAGCAATGCTT G. max 1418 CCTCTGGTTCCACCGGGTACACCAGTGGTGAATGCATTGTCAAAGCAGCGTGCAATGCTG

\begin{tabular}{|c|c|c|}
\hline $1-150-81$ & 1499 & GAAAACATCATGAGAGCTTGTGTTGGATTGGCTCCAGAAAACAACATGATCCTTGAGTAC \\
\hline$-2347-144$ & 1500 & GAAAACATCATGAGAGCTTGTGTTGGATTGGCTCCAGAAAACAACATGATCCTTGAGTAC \\
\hline truncatula & 1468 & GAAAACATCATGAGAGCTTGTGTTGGATTGGCTCCAGAGAACAACATGATCCTCGAGTAC \\
\hline & 1478 & GAAAACATAAGAGGGCTTGTGTTGGATTGGCDCCAGAGAATAACATGATTCTCGAGTA \\
\hline
\end{tabular}

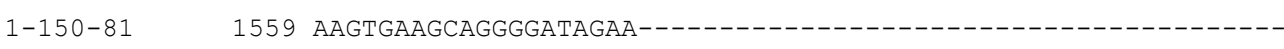

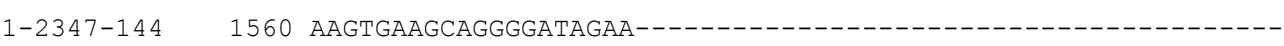

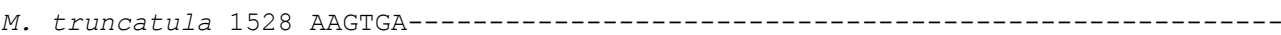
G. max 1538 AAGTGAAGCATGGGACCGAAGAATAATATAGTTGGGGTAGCCTAGCTGAATGTTTTATGT

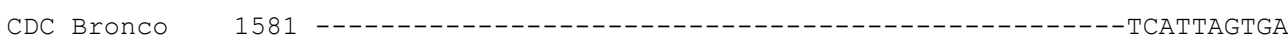

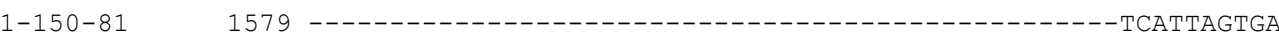
1-2347-144 1580 M. truncatula G. $\max$ 1598 TAATAATATGTTTGCTTATAATTTTGCAAGTGTAATTGAATGCATCAGCTTCATTAATGC

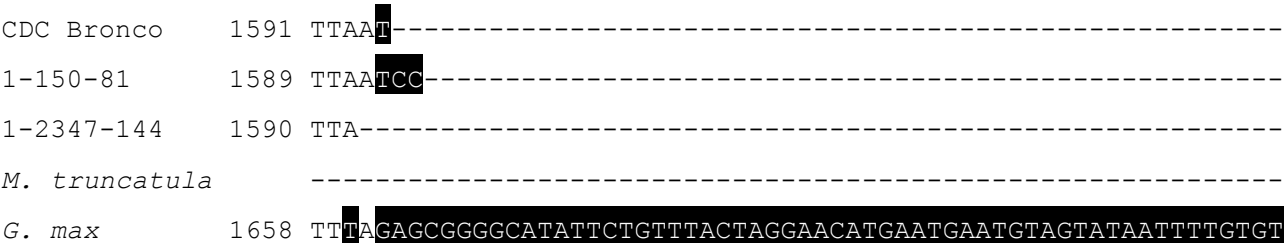




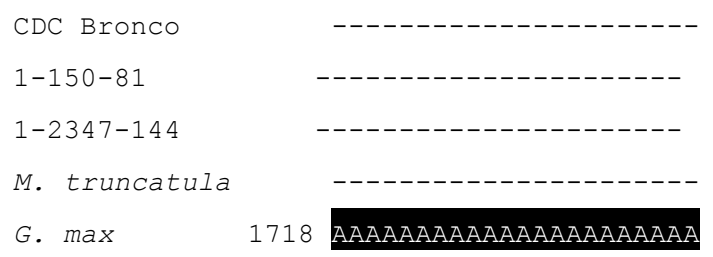

Figure A2. Nucleotide sequence alignment of PSMIPS for CDC Bronco and low-phytate genotypes 1-150-81 and 1-2347-144. Also included for comparison are nucleotide sequences for MIPS obtained from GenBank for Medicago truncatula (XM_003601939.1) and Glycine max (AY038802.1). Nucleotide differences are indicated in black boxes.

\section{Conflicts of Interest}

The authors declare no conflict of interest.

\section{References}

1. Cosgrove, D.J. Inositol Phosphates: Their Chemistry, Biochemistry, and Physiology; Elsevier: New York, NY, USA, 1980.

2. O’Dell, B.L.; de Boland, A.R.; Koirtyohann, S.R. Distribution of phytate and nutritionally important elements among the morphological components of cereal grains. J. Agric. Food. Chem. 1972, 20, 718-721.

3. Lott, J.N.A. Accumulation of seed reserves of phosphorus and other minerals. In Seed Physiology; Murray, D.R., Eds.; Academic Press: Sydney, Australia, 1984; Volume 1, pp. 154-196.

4. Raboy, V. Seeds for a better future: "Low phytate" grains help to overcome malnutrition and reduce pollution. Trends Plant Sci. 2001, 6, 458-462.

5. Reddy, N.R. Occurrence, distribution, content and dietary intake of phytate. In Food Phytates; Reddy, N.R., Sathe, S.K., Eds.; CRC Press: New York, NY, USA, 2002; pp. 25-51.

6. Erdman, J.W., Jr. Bioavailability of trace minerals from cereals and legumes. Cereal Chem. 1980, $58,21-26$.

7. Sharpley, A.N.; Charpa, S.C.; Wedepohl, R.; Sims, J.Y.; Daniel, T.C.; Reddy, K.R. Managing agricultural phosphorus for protection of surface waters: Issues and Options. J. Environ. Qual. 1994, 23, 437-451.

8. Raboy, V.; Young, K.A.; Dorsch, J.A.; Cook, A. Genetics and breeding of seed phosphorus and phytic acid. J. Plant Physiol. 2001, 158, 489-497.

9. Raboy, V.; Gerbasi, P. Genetics of myo-inositol phosphate synthesis and accumulation. In Myo Inositol Phosphates, Phosphoinositides and Signal Transduction; Biswas, B.B., Ed.; Plenum Publ. Co.: New York, NY, USA, 1996; pp. 257-285.

10. Raboy, V.; Gerbasi, P.F.; Young, K.A.; Stoneberg, S.D.; Pickett, S.G.; Bauman, A.T.; Murthy, P.N.; Sheridan, W.F.; Ertl, D.S. Origin and seed phenotype of maize low phytic acid $1-1$ and low phytic acid 2-1. Plant Physiol. 2000, 124, 355-368.

11. Larson, S.R.; Young, K.A.; Cook, A.; Blake, T.K.; Raboy, V. Linkage mapping of two mutations that reduce the phytic acid content of barley grain. Theor. Appl. Genet. 1998, 97, 141-146. 
12. Rasmussen, S.K.; Hatzack, F. Identification of two low-phytate barley (Hordeum vulgare L.) grain mutants by TLC and genetic analysis. Heriditas 1998, 129, 107-112.

13. Wilcox, J.; Premachandra, G.; Young, K.; Raboy, V. Isolation of high seed inorganic P, low-phytate soybean mutants. Crop Sci. 2000, 40, 1601-1605.

14. Warkentin, T.D.; Delgerjav, T.; Arganosa, G.; Rehman, A.U.; Bett, K.E.; Anbessa, Y.K.; Rossnagel, B.; Raboy, V. Development and characterization of low-phytate pea. Crop Sci. 2012, $52,74-78$.

15. Ertl, D.S.; Young, K.A.; Raboy, V. Plant genetic approaches to phosphorus management in agricultural production. J. Environ. Qual. 1998, 27, 299-304.

16. Loewus, F.A.; Murthy, P.N. Myo-inositol metabolism in plants. Plant Sci. 2000, 150, 1-19.

17. Stevenson, J.M.; Perera, I.Y.; Heilmann, I.; Persson, S.; Boss, W.F. Inositol signaling and plant growth. Trends Plant Sci. 2000, 5, 252-258.

18. Loewus, F.A.; Loewus, M.W. Myo-inositol: Its biosynthesis and metabolism. Annu. Rev. Plant Physiol. 1983, 34, 137-161.

19. Loewus, F.A. Inositol biosynthesis. In Inositol Metabolism in Plants; Morre, D.J., Boss, W.F., Loewus, F.A., Eds.; Wiley-Liss: New York, NY, USA, 1990; pp. 13-19.

20. Pilu, R.; Panzeri, D.; Gavazzi, G.; Rasmussen, S.K.; Consonni, G.; Nielsen, E. Phenotypic, genetic and molecular characterization of a maize low phytic acid mutant (lpa241). Theor. Appl. Genet. 2003, 107, 980-987.

21. Kuwano, M.; Ohyama, A.; Tanaka, Y.; Mimura, T.; Takaiwa, F.; Yoshida, K.T. Molecular breeding for transgenic rice with low-phytic-acid phenotype through manipulating myo-inositol 3-phosphate synthase gene. Mol. Breed. 2006, 18, 263-272.

22. Kuwano, M.; Mimura, T.; Takaiwa, F. Generation of stable "low phytic acid" transgenic rice through antisense repression of the 1d-myo-inositol 3-phosphate synthase gene (RINO1) using the 18-kDa oleosin promoter. Plant Biotech. J. 2009, 7, 96-105.

23. Georges, F.; Hussain, A.A.K.; Keller, W.A. Method for Reducing Phytate in Canola Meal Using Genetic Manipulation Involving Myo-Inositol 1-Phosphate Synthase Gene. Patent WO 00/73473 A1, 2006.

24. Nunes, A.C.S.; Vianna, G.R.; Cuneo, F.; Amaya-Farfán, J.; de Capdeville, G.; Rech, E.L.; Aragão, F.J.L. RNAi-mediated silencing of the myo-inositol-1-phosphate synthase gene (GmMIPS1) in transgenic soybean inhibited seed development and reduced phytate content. Planta 2006, 224, 125-132.

25. Panzeri, D.; Cassani, E.; Doria, E.; Tagliabue, G.; Forti, L.; Campion, B.; Bollini, R.; Brearley, C.A.; Pilu, R.; Nielsen, E.; et al. A defective ABC transporter of the MRP family, responsible for the bean lpa 1 mutation, affects the regulation of the phytic acid pathway, reduces seed myo-inositol and alters ABA sensitivity. New Phytol. 2011, 191, 70-83.

26. Hitz, D.W.; Thomas, J.C.; Phil, S.K.; Scott, A.S. Biochemical and molecular characterization of a mutation that confers a decreased raffinosaccharide and phytic acid phenotype on soybean seeds. Plant Physiol. 2002, 128, 650-660.

27. Shunmugam, A.S.K.; Bock, C.; Arganosa, G.C.; Gray, R.G.; Georges, F.; Warkentin, T.D. Department of Plant Sciences, University of Saskatchewan: Saskatoon, Saskatchewan, Canada; Unpublished raw data, 2014. 
28. Majumder, A.L.; Johnson, M.D.; Henry, S.A. 1L-myo-inositol-1-phosphate synthase. Biochim. Biophys. Acta 1997, 1348, 245-256.

29. Majumder, A.L.; Chatterjee, A.; Dastida, K.G.; Majee, M. Diversification and evolution of L-myo-inositol 1-phosphate synthase. FEBS Lett. 2003, 553, 3-10.

30. Gillman, J.D.; Pantalone, V.R.; Bilyeu, K. The low phytic acid phenotype in soybean line CX1834 is due to mutations in two homologs of the maize low phytic acid gene. Plant Genome 2009, 2, 179-190.

31. Anderson, B.P.; Fehr, W.R. Seed source affects field emergence of low-phytate soybean lines. Crop Sci. 2008, 48, 929-932.

32. Bentsink, K.; Yuan, Y.; Koorneef, M.; Vreugdenhil, D. The genetics of phytate and phosphorus accumulation in seeds and leaves of Arabidopsis thaliana, using natural variation. Theor. Appl. Genet. 2003, 106, 1234-1243.

33. Shi, J.R.; Wang, H.Y.; Schellin, K.; Li, B.L.; Faller, M.; Stoop, J.M.; Meeley, R.B.; Ertl, D.S.; Ranch, J.P.; Glassman, K. Embryo-specific silencing of a transporter reduces phytic acid content of maize and soybean seeds. Nat. Biotechnol. 2007, 25, 930-937.

34. Dong, J.; Yan, W.; Bock, C.; Nokhrina, K.; Keller, W.; Georges, F. Perturbing the metabolic dynamics of myo-inositol in developing Brassica napus seeds through in vivo methylation impacts its utilization as phytate precursor and affects downstream metabolic pathways. BMC Plant Biol. 2013, 13, e84.

35. Ali, N.; Paul, S.; Gayen, D.; Sarkar, S.N.; Datta, K.; Datta, S.K. Development of low phytate rice by RNAi mediated seed-specific silencing of inositol 1,3,4,5,6-pentakisphosphate 2-kinase gene (IPK1). PLOS ONE 2013, doi:10.1371/journal.pone.0068161.

36. Bregitzer, P.; Raboy, V. Effects of four independent low-phytate mutations on barley agronomic performance. Crop Sci. 2006, 46, 1318-1322.

37. Bregitzer, P.; Raboy, V.; Obert, D.E.; Windes, J.; Whitmore, J.C. Registration of "Clearwater" low-phytate hulless spring barley. J. Plant Registr. 2008, 2, 1-4.

38. Raboy, V.; Dikinson, D.B.; Neuffer, M.G. A survey of maize kernel mutants for variation in phytic acid. Maydica 1990, 35, 383-390.

39. Raboy, V. Low Phytic acid Mutants and Selection Thereof. Patent 5,689,054, 1997.

40. Raboy, V.; Dickinson, D.B. The timing and rate of phytic acid accumulation in developing soybean seeds. Plant Physiol. 1987, 85, 0841-0844.

41. Bowen, D.E.; Guttieri, M.J.; Peterson, K.; Peterson, K.; Raboy, V.; Souza, E.J. Phosphorus fractions in developing seeds of four low phytate barley (Hordeum vulgare L.) genotypes. Crop Sci. 2006, 46, 2468-2473.

42. Israel, D.W.; Talierico, E.; Kwanyuen, P.; Burton, J.W.; Dean, L. Inositol metabolism in developing seeds of low and normal phytic acid soybean lines. Crop Sci. 2011, 51, 282-289.

43. Larson, S.R.; Rutger, J.N.; Young, K.A.; Raboy, V. Isolation and genetic mapping of a non-lethal rice (Oryza sativa L.) low phytic acid 1 mutation. Crop Sci. 2000, 40, 1397-1405.

44. Yoshida, K.T.; Wada, T.; Koyama, H.; Mizobuchi-Fukuoka, R.; Naito, S. Temporal and spatial patterns of accumulation of the transcript of myo-inositol-1-phosphate synthase and phytin-containing particles during seed development in rice. Plant Physiol. 1999, 119, 65-72. 
45. Ma, D.; Zuo, Y.; Sun, D.; Wang, C.; Guo, T. Characterization of the TaMIPS gene from winter wheat (Triticum aestivum L.) and changes in its expression pattern with phytic acid accumulation in seeds during grain filling. J. Cereal Sci. 2013, 57, 437-443.

46. Keller, R.; Brearley, C.A.; Trethewey, R.N.; Muller-Rober, B. Reduced inositol content and altered morphology in transgenic potato plants inhibited for 1D-myo-inositol 3-phosphate synthase. Plant J. 1998, 16, 403-410.

47. Murphy, A.M.; Otto, B.; Brearley, C.A.; Carr, J.P.; Hanke, D.E. A role for inositol hexakisphosphate in the maintenance of basal resistance to plant pathogens. Plant J. 2008, 56, 638-652.

48. Xu, X.H.; Zhao, H.J.; Liu, Q.L.; Frank, T.; Engel, K.H.; An, G.H.; Shu, Q.Y. Mutations of the multi-drug resistance-associated protein $\mathrm{ABC}$ transporter gene 5 result in reduction of phytic acid in rice seeds. Theor. Appl. Genet. 2009, 119, 75-83.

49. Yuan, F.J.; Zhao, H.J.; Ren, X.L.; Zhu, S.L.; Fu, X.J.; Shu, Q.Y. Generation and characterization of two novel low phytate mutations in soybean (Glycine max L. Merr.). Theor. Appl. Genet. 2007, $115,945-957$.

50. Shi, J.; Wang, H.; Wu, Y.; Hazebroek, J.; Meeley, R.B.; Ertl, D.S. The maize low-phytic acid mutant lpa2 is caused by mutation in an inositol phosphate kinase gene. Plant Physiol. 2003, 131, 507-515.

51. Stevenson-Paulik, J.; Bastidas, R.J.; Chiou, S.T.; Frye, R.A.; York, J.D. Generation of phytate-free seeds in Arabidopsis through disruption of inositol polyphosphate kinases. Proc. Natl. Acad. Sci. USA 2005, 102, 12612-12617.

52. Warkentin, T.D.; Vandenberg, A.; Banniza, S.; Slinkard, A. CDC Bronco field pea. Can. J. Plant Sci. 2005, 85, 649-650.

53. Talamond, P.; Doulbeau, S.; Rochette, I.; Guyot, J.P. Anion exchange high-performance liquid chromatography with conductivity detection for the analysis of phytic acid in food. J. Chromatogr. A 2000, 871, 7-12.

54. Thavarajah, P.; Thavarajah, D.; Vandenberg, A. Low phytic acid lentils (Lens culinaris L.): A potential solution for increased micronutrient bioavailability. J. Agric. Food Chem. 2009, 57, 9044-9049.

55. Chen, P.S.; Toribara, T.Y.; Warner, H. Microdetermination of phosphorus. Anal. Chem. 1956, 28, $1756-1758$.

56. Main, D.; Cheng, C-H.; Ficklin, S.P.; Jung, S.; Zheng, P.; Coyne, C.J.; McGee, R.; Mockaitis, K. In The Cool Season Food Legume Database: An Integrated Resource for Basic, Translational and Applied Research, Proceedings of the International Plant and Animal Genome Conference, San Diego, CA, USA, 12-16 January 2013.

57. Ye, J.; Coulouris, G.; Zaretskaya, I.; Cutcutache, I.; Rozen, S.; Madden, T. Primer-BLAST: A tool to design target-specific primers for polymerase chain reaction. BMC Bioinform. 2012, 13, e134.

58. Sambrook, J.; Russell, D.W. Molecular Cloning, A Laboratory Manual, 3rd ed.; Cold Spring Harbour Laboratory Press: Cold Spring Harbour, New York, NY, USA, 2001; Volume 1-3.

59. Ozga, J.A.; Yu, J.; Reinecke, D.M. Pollination-, development-, and auxin-specific regulation of gibberellin 3 $\beta$-hydroxylase gene expression in pea fruit and seeds. Plant Physiol. 2003, 131, 1137-1146.

60. Hall, T.A. BioEdit: A user-friendly biological sequence alignment editor and analysis program for Windows 95/98/NT. Nucl. Acids Symp. Ser. 1999, 41, 95-98. 
61. Altschul, S.F.; Madden, T.L.; Schaffer, A.A.; Zhang, J.; Zhang, Z.; Miller, W.; Lipman, D.J. Gapped Blast and Psi-Blast: A new generation of protein database search programs. Nucleic Acids Res. 1997, 25, 3389-3402.

62. Larkin, M.A.; Blackshields, G.; Brown, N.P.; Chenna, R.; McGettigan, P.A.; McWilliam, H.; Valentin, F.; Wallace, I.M.; Wilm, A.; Lopez, R.; et al. Clustal W and Clustal X version 2.0. Bioinformatics 2007, 23, 2947-2948.

63. Quevillon, E.; Silventoinen, V.; Pillai, S.; Harte, N.; Mulder, N.; Apweiler, R.; Lopez, R. InterProScan: Protein domains identifier. Nucleic Acids Res. 2005, 33, 116-120.

64. Tamura, K.; Stecher, G.; Peterson, D.; Filipski, A.; Kumar, S. MEGA6: Molecular Evolutionary Genetics Analysis version 6.0. Mol. Biol. Evol. 2013, 30, 2725-2729.

(C) 2014 by the authors; licensee MDPI, Basel, Switzerland. This article is an open access article distributed under the terms and conditions of the Creative Commons Attribution license (http://creativecommons.org/licenses/by/4.0/). 